\title{
SREBP1 silencing inhibits the proliferation and motility of human esophageal squamous carcinoma cells via the Wnt/ק-catenin signaling pathway
}

\author{
JINGZHI WANG $^{1 *}$, RUI LING $^{1 *}$, YUEPENG ZHOU $^{1}$, XINGYU GAO $^{1}$,

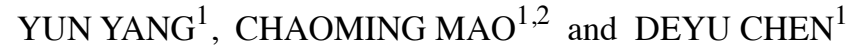 \\ ${ }^{1}$ Institute of Oncology; ${ }^{2}$ Department of Nuclear Medicine, Affiliated Hospital of Jiangsu University, \\ Zhenjiang, Jiangsu 212001, P.R. China
}

Received September 10, 2019; Accepted May 22, 2020

DOI: 10.3892/ol.2020.11853

\begin{abstract}
Sterol regulatory element-binding protein 1 (SREBP1) is dysregulated in a variety of types of human cancer. However, the functional roles of SREBP1 in esophageal squamous cell carcinoma (ESCC) remain poorly understood. The present study investigated the function of SREBP1 in cell proliferation and motility. Microarray datasets in Oncomine, reverse transcription-quantitative PCR and western blot analysis revealed that SREBP1 was overexpressed in ESCC tumors when compared with normal tissues. In addition, SREBP1 overexpression was significantly associated with tumor differentiation, lymphatic metastasis and Ki67 expression. Results suggested that silencing SREBP1 inhibited the proliferation, migration and invasion of ESCC cells, whereas overexpression of SREBP1 had opposite effects on proliferation and metastasis. In addition, loss of SREBP1 significantly increased E-cadherin and decreased $\mathrm{N}$-cadherin, Vimentin, Snail, matrix metalloproteinase 9 and vascular endothelial growth factor $\mathrm{C}$ expression levels, which were restored via SREBP1-overexpression. Mechanistically, loss of SREBP1 suppressed T-cell factor $1 /$ lymphoid enhancer factor 1 (TCF1/LEF1) activity and downregulated TCF1/LEF1 target proteins, including CD44 and cyclin D1. Moreover, knockdown of SREBP1 downregulated the expression levels of stearoyl-CoA desaturase 1 (SCD1), phosphorylated glycogen synthase kinase- $3 \beta$ and nuclear $\beta$-catenin. Furthermore, the inhibitors of SREBP1 and/or SCD1 and small interfering RNA-SCD1
\end{abstract}

Correspondence to: Dr Deyu Chen or Mr. Yuepeng Zhou, Institute of Oncology, Affiliated Hospital of Jiangsu University, 438 Jiefang Road, Zhenjiang, Jiangsu 212001, P.R. China

E-mail: cdeyu@hotmail.com

E-mail: yuepeng_zhou@ujs.edu.cn

*Contributed equally

Key words: sterol regulatory element-binding protein 1, esophageal squamous cell carcinoma, proliferation, motility, Wnt/ $\beta$-catenin efficiently inhibited the activation of the Wnt/ $\beta$-catenin pathway driven by constitutively active SREBP1. Finally, in vivo results indicated that SREBP1-knockdown suppressed the proliferation and metastasis of ESCC. Taken together, these findings demonstrated that SREBP1 exerts oncogenic effects in ESCC by promoting proliferation and inducing epithelial-mesenchymal transition via the SCD1-induced activation of the Wnt/ $\beta$-catenin signaling pathway.

\section{Introduction}

Esophageal cancer is a common and refractory type of gastrointestinal cancer worldwide, and ranks 9th in the incidence rate of all cancer types and has the 6th highest malignant tumor-associated mortality rate, with $\sim 572,000$ new worldwide cases and more than half a million cancer-associated deaths in 2018 (1). Of all reported esophageal cancer subtypes, $\sim 90 \%$ are esophageal squamous cell carcinoma (ESCC) globally each year, which is usually present at an advanced stage and is mostly concentrated in China $(2,3)$. As many as $90 \%$ of patients with ESCC died from distant invasion and metastasis and $36.8 \%$ of patients had lymph node metastasis between January 2010 and July 2016 in China $(4,5)$. Therefore, the molecular mechanisms underlying tumor invasion and metastasis must be investigated to develop novel antineoplastic strategies for metastatic ESCC treatment.

Metastasis is a dynamic process in which cancer cells spread from the original niche to neighbor and/or distant tissues by acquiring malignant capabilities (6). In metastatic ESCC, malignant cells often maintain their mesenchymal characteristics with reduced E-cadherin and increased Vimentin, N-cadherin, matrix metalloproteinase (MMP) and vascular endothelial growth factor (VEGF) expression levels, forming the epithelial-mesenchymal transition (EMT) and other aggressive phenotypes (7-9). Various signaling cascades contribute to the onset of EMT and further metastasis, including the $\mathrm{Wnt} / \beta$-catenin pathway (8-12). It has previously been reported that aberrant $\mathrm{Wnt} / \beta$-catenin signaling promotes EMT and metastasis in ESCC (7,11). Furthermore, proliferating cancer cells require extensive synthesis or uptake of lipids to form cytoplasmic organelles, plasma membranes or other 
lipid conformations $(13,14)$. Thus, the regulators and pathways associated with cancer lipid metabolism need to be elucidated. Several studies have implicated that stearoyl-CoA desaturase 1 (SCD1), which primarily converts saturated fatty acids into monounsaturated fatty acids, inhibits glycogen synthase kinase- $3 \beta$ (GSK-3 $\beta$ ) to degrade $\beta$-catenin and promote T-cell factor 1/lymphoid enhancer factor 1 (TCF1/LEF1) activity in tumors (15-18). However, whether SCD1 contributes to Wnt/ $\beta$-catenin signaling activation in ESCC cells or how the upregulation of SCD1 expression levels is established in tumor proliferation, EMT and metastases is unclear.

Given the specific roles of sterol regulatory element-binding proteins (SREBPs) as transcription factors and functions as primary regulatory elements of sterol biosynthesis and lipid metabolism, SREBPs are increasingly being viewed as major regulatory molecules for signaling events (19-22). Mammalian genomes have two SREBP genes, SREBP1 and SREBP2, both of which are key regulators of nutritional homeostasis (22). SREBP1 is involved in cancer aggressiveness and is associated with clinical status and a less favorable prognosis in different types of tumor (23-26). SREBP1-mediated regulation of fatty acid and lipid synthesis is implicated in the pathogenesis of prostate cancer, and SREBP1 promotes the invasion and metastasis of colorectal cells by regulating the expression of MMP7 (27,28). Moreover, Wnt/ $\beta$-catenin signaling can upregulate the expression levels of SREBP1 in sebaceous gland cells in three-dimensional culture, and SREBP1 and $\beta$-catenin interaction is as a vital component of the proliferation and metastasis of colorectal neoplasm cells $(29,30)$. Consistent with its function as an oncogene, SREBP1 is activated and cleaved from full length SREBP1 (125 kDa), which is retained in the endoplasmic reticulum, to a soluble active fragment mature (m)SREBP1 $(68 \mathrm{kDa})$, which is translocated into the nucleus where it functions as a transcription factor of lipid synthesis genes, including SCD1 (31,32). As mediators of the Hippo pathway, Yes-associated protein/transcription coactivator with PDZ-binding motif activation is promoted by increased levels of mevalonic acid produced by SREBP1 in tumor cells, and SREBP1-dependent lipid anabolism is prevented by extracellular matrix stiffening and geranylgeranylated RhoA-dependent acto-myosin contraction via activation of the AMP-activated protein kinase (AMPK) pathway $(31,32)$. This suggests that SREBP1 has an evolutionary conserved function in rewiring cell metabolism in response to metabolic and mechanical cues $(31,32)$. Although SREBP1 may serve a role in tumorigenesis, most studies have focused on the association of lipid metabolism in cancer cells (25,33-36). The molecular mechanism of SREBP1 and SCD1 in the regulation of the Wnt pathway and ESCC progression, especially in EMT, migration and invasion, has not yet been investigated.

The present study aimed to explore the role of SREBP1/SCD1 in regulation of proliferation, invasion and metastasis in ESCC. SREBP1/SCD1 may serve as potential therapeutic targets in ESCC.

\section{Materials and methods}

Oncomine database analysis. Two primary sources for the ESCC data were obtained from Oncomine database (https://www.oncomine.org) $(37,38)$ to determine the mRNA expression levels of SREBP1. The two groups had sample sizes of 56 and 17 cases, respectively. Median age, sex and age range data were not available in the Oncomine database.

Immunohistochemical (IHC) analysis. Tissue samples were collected during surgical resection after informed written consent had been provided by patients with ESCC (Table I). The patients were diagnosed by two pathologists in accordance with the World Health Organization classification without chemotherapy or radiotherapy before resection, as described previously (39). The 77 paired ESCC tumor and adjacent normal tissue samples that were $2 \mathrm{~cm}$ away from the tumors' edge, were collected between February 2018 and July 2019 at the Affiliated Hospital of Jiangsu University (Zhenjiang, China). The IHC staining procedure used to determine SREBP1 expression levels was performed as previously described (7,39). The sections were incubated with antibody against SREBP1 (1:100; catalog no. 14088-1-AP; Proteintech Group, Inc.) overnight at $4^{\circ} \mathrm{C}$. Briefly, slides were evaluated at a magnification of $\mathrm{x} 200$ and 5 representative fields of each section were assessed independently by 2 trained observers who were blinded to patient information. Identical settings were used for all of the images. The integrated absorbance and area of the images were counted by Image-Pro Plus version 6.0 software (Media Cybernetics, Inc.), and the sections were classified as either high or low expression. The intensity of staining was scored as follows: 0 , no staining; 1 , weak staining; 2 , moderate staining; and 3, strong staining. The proportion of stained cells was scored as follows: 0 , no cells stained; 1 , $<50 \%$ of cells stained positive; 2,50 to $75 \%$ of cells stained positive; and $3,>75 \%$ of cells stained positive. The final score was determined by combining the 2 scores. A score of $\leq 6$ was considered low expression, and a score $>6$ was considered high expression. The present study was approved by The Medical Ethical Committee of Affiliated Hospital of Jiangsu University (Zhenjiang, China).

Cell culture and transfection. ESCC cell lines TE-1, ECA-109 and KYSE-150 were purchased from Shanghai GeneChem Co., Ltd., which were authenticated using Short Tandem Repeat profiling. All experiments using these cell lines were performed within 6 months of receipt or thawing after $-80^{\circ} \mathrm{C}$ cryopreservation. ESCCs were cultured in RPMI-1640 (Gibco; Thermo Fisher Scientific, Inc.) supplemented with $10 \%$ fetal bovine serum (FBS; Biological Industries) and $1 \%$ penicillin-streptomycin (Gibco; Thermo Fisher Scientific, Inc.) at $37^{\circ} \mathrm{C}$ in a thermostatic incubator containing $5 \% \mathrm{CO}_{2}$. Immortalized human normal esophageal epithelial cells, Het-1A (BeNa Culture Collection), were maintained in DMEM (Gibco; Thermo Fisher Scientific, Inc.) supplemented with $10 \%$ FBS and containing $1 \%$ penicillin-streptomycin, as described previously (39). Wnt/ $\beta$-catenin inhibitor ICG-001 was obtained from Selleck Chemicals and dissolved in dimethyl sulfoxide as previously described $(7,40)$. Fatostatin (inhibitor of SREBP) and Mevastatin [an inhibitor of HMG-CoA reductase (HMGCR)] were purchased from MedChemExpress and dissolved in dimethyl sulfoxide. ESCC cells received treatment with ICG-001, Fatostatin and Mevastatin at the recommended concentration of $10 \mu \mathrm{M}$ at $37^{\circ} \mathrm{C}$ for $48 \mathrm{~h}(41,42)$.

Since ECA-109 andKYSE-150 are classic cell lines of human esophageal squamous cell carcinoma and these 2 cell lines are 
Table I. Association of SREBP1 expression levels with clinicopathological features of 77 patients with esophageal squamous cell carcinoma.

SREBP1 expression

\begin{tabular}{|c|c|c|c|c|}
\hline \multirow{2}{*}{ Clinicopathological feature } & \multirow[b]{2}{*}{$\mathrm{n}$} & & \multirow[b]{2}{*}{ P-value } \\
\hline & & High & Low & \\
\hline Sex & & & & 0.2832 \\
\hline Male & 51 & 19 & 32 & \\
\hline Female & 26 & 13 & 13 & \\
\hline Age, years & & & & 0.7929 \\
\hline$\leq 61$ & 18 & 7 & 11 & \\
\hline$>61$ & 59 & 25 & 34 & \\
\hline Diameter of tumor, $\mathrm{cm}$ & & & & 0.1309 \\
\hline$>5$ & 24 & 13 & 11 & \\
\hline$\leq 5$ & 53 & 19 & 34 & \\
\hline Tumor location & & & & 0.0939 \\
\hline Upper/middle & 32 & 16 & 16 & \\
\hline Lower & 45 & 14 & 31 & \\
\hline Tumor stage & & & & 0.1974 \\
\hline I-II & 52 & 19 & 33 & \\
\hline III-IV & 25 & 13 & 12 & \\
\hline Degree of differentiation & & & & 0.0137 \\
\hline Well & 24 & 5 & 19 & \\
\hline Moderately & 29 & 12 & 17 & \\
\hline Poorly & 24 & 15 & 9 & \\
\hline Lymph node metastasis & & & & $<0.0001$ \\
\hline Yes & 35 & 27 & 8 & \\
\hline No & 42 & 5 & 37 & \\
\hline Ki-67 expression, $\%$ & & & & 0.0004 \\
\hline$\leq 50$ & 40 & 9 & 31 & \\
\hline$>50$ & 37 & 23 & 14 & \\
\hline
\end{tabular}

SREBP1, sterol regulatory element-binding protein 1 (61).

stable after lentivirus transfection, which is convenient for cell sub-culture (43). ECA-109 and KYSE-150 cells were chosen for transfection experiments in the present study. Lentiviral vectors with small hairpin (sh)RNA-SREBP1 (shSREBP1 Bank Id: NM_004176, NM_001321096 and NM_001005291), SREBP1-expressing vector (Bank Id NM_001321096), empty vector control and scrambled control shRNA as negative control (shNC) were purchased from Shanghai GeneChem Co., Ltd. The cell concentration was adjusted to $5 \times 10^{4}$ cells $/ \mathrm{ml}$ and inoculated in 6-well plate with $2 \mathrm{ml} /$ well of culture medium. After $24 \mathrm{~h}$ of cell adherence, culture medium was replaced by serum-free medium $(1 \mathrm{ml})$ and then $20 \mu \mathrm{l}$ lentiviral vectors, shRNA and controls $\left(1 \times 10^{8} \mathrm{TU} / \mathrm{ml}\right)$ and $4 \mu \mathrm{l}$ of HitransG A (Shanghai GeneChem Co., Ltd.) were added. The efficiency of transfection was verified via western blotting $72 \mathrm{~h}$ following transfection and subsequent experiments were performed. The concentration of shRNA, vector and controls transfected are according to the manufacturer's protocol. The transfection reagent used was HitransG A (Shanghai GeneChem Co., Ltd.). The small interfering (si)RNA against NC, SREBP1 and SCD1 were designed and synthesized by Shanghai GenePharma Co., Ltd. In order to assess the effects of short- and long-term silencing of SREBP1, shSREBP1 was used to silence SREBP1 for a long time and siSREBP1was used to silence SREBP1 for a short time. The siRNA sequences were as follows: NC, 5'-GCGACGAUCUGCCUAAGAU-3'; SREBP1, 5'-GCU CCUCACUUGAAGGCUUTT-3'; and SCD1, 5'-TGAAAG AAGATATTCACGA-3'. siRNA (40 nM) was transfected using Lipofectamine ${ }^{\circledR} 2000$ (Thermo Fisher Scientific, Inc.) in serum-free conditions for $7 \mathrm{~h}$ prior to replacement with complete medium. The efficiency of silencing or overexpression were confirmed by western blotting.

Reverse transcription-quantitative $(R T-q) P C R$.mRNA expression levels in the clinical specimens of paired ESCC and normal control tissues were compared. In total, 20 pairs of frozen ESCC tumors and matched normal tissues were randomly selected, immediately snap-frozen in liquid nitrogen and stored at $-80^{\circ} \mathrm{C}$. RNA was extracted from these tissues using RNAiso Plus (Takara Biotechnology Co., Ltd.), in accordance with the 
manufacturer's instructions, and prepared for RT into cDNA using a reverse transcription reagent kit (cat. no. R123-01; Vazyme Biotech Co., Ltd). qPCR was performed using a PCR kit (cat. no. Q311-02/03; Vazyme Biotech Co., Ltd.), and the primers used were synthesized by Invitrogen; Thermo Fisher Scientific, Inc. The qPCR thermocycling conditions were as follows: 1 cycle at $95^{\circ} \mathrm{C}$ for $30 \mathrm{sec} ; 40$ cycles at $95^{\circ} \mathrm{C}$ for $10 \mathrm{sec}$, $60^{\circ} \mathrm{C}$ for $30 \mathrm{sec}$ and $3 \mathrm{cycles}$ at $95^{\circ} \mathrm{C}$ for $15 \mathrm{sec}, 60^{\circ} \mathrm{C}$ for $60 \mathrm{sec}$, $95^{\circ} \mathrm{C}$ for $15 \mathrm{sec}$ following the protocols of ChamQ ${ }^{\mathrm{TM}} \mathrm{SYBR}$ qPCR Master Mix (Vazyme Biotech Co. Ltd.). The sequences were as follows: SREBP1 forward, 5'-CGGCGCTGCTGA CCGACATC-3' and reverse, 5'-CCCTGCCCCACTCCCAGC AT-3'; Snaill forward, 5'-CTTCTCCTCTACTTCAGTCTC TTCC-3' and reverse, 5'-TGAGGTATTCCTTGTTGCAGT ATTT-3'; Slug forward, 5'-AACAGAGCATTTGCAGACAGG TC-3' and reverse, 5'-GCTACACAGCAGCCAGATTCC-3'; MMP2 forward, 5'-CTTCCAAGTCTGGAGCGATGT-3' and reverse, 5'-TACCGTCAAAGGGGTATCCAT-3'; MMP9 forward, 5'-GGGACGCAGACATCGTCATC-3' and reverse, 5'-TCGTCATCGTCGAAATGGGC-3'; VEGF-A forward, 5'-AGGAGGAGGGCAGAATCATCA-3' and reverse, 5'-CTC ATTGGATGGCAGTAGCT-3'; VEGF-C, forward, 5'-TGT GTGTCCGTCTACAGATGTG-3' and reverse, 5'-TCGGCA GGAAGTGTGATTGG-3'; and GAPDH forward, 5'-AGC CACATCGCTCAGACAC-3' and reverse, 5'-GCCCAATAC GACCAAATCC-3'. Relative expression levels were calculated using the $2^{-\Delta \Delta \mathrm{Cq}}$ method and GAPDH was used as an internal reference gene (44).

Western blotting. The total cellular or tissue protein was extracted using RIPA lysis buffer (Beijing Solarbio Science \& Technology Co., Ltd.) at $4^{\circ} \mathrm{C}$. Cytoplasmic protein and nuclear proteins were extracted using the Cytoplasmic Protein Extraction kit (cat. no. BC3740; Beijing Solarbio Science \& Technology Co., Ltd.) and Nuclear Protein Extraction kit (cat. no. R0050; Beijing Solarbio Science \& Technology Co., Ltd.) according to the manufaturer's protocols. Then, protein concentration was calculated using a BCA protein assay kit (CoWin Biosciences). Following that, equivalent amounts of total protein (40 $\mu \mathrm{g} /$ lane) were added into each lane of $8-15 \%$ gel, resolved using SDS-PAGE and transferred to PVDF membranes. Membranes were blocked using 5\% bovine serum albumin (BSA) or non-fat milk diluted in TBST for $1 \mathrm{~h}$ at room temperature. Thereafter, the blocked membranes were incubated with the following primary antibodies at $4^{\circ} \mathrm{C}$ overnight: SREBP1 (1:1,000; catalog no. sc-13551; Santa Cruz Biotechnology, Inc., or 1:1,000; catalog no. 14088-1-AP; Proteintech Group, Inc. Two different antibodies were used to ensure the specificity of antibodies and results), SREBP2 (1:1,000; cat. no. sc-13552; Santa Cruz Biotechnology, Inc.), SCD1 (1:1,000; catalog no. ab236868); GSK-3 $\beta$ (1:1,000; catalog no. ab68476), phosphorylated (p)-GSK-3 $\beta$ (1:1,000; catalog no. ab32391), VEGF-A (1:1,000; catalog no. ab1316), MMP2 (1:1,000; catalog no. ab92536), MMP9 (1:1,000; catalog no. ab76003; Abcam), E-cadherin $(1: 1,000$; catalog no. 14472), Vimentin (1:1,000; catalog no. 5741), Snail (1:1,000; catalog no. 3879), $\beta$-catenin $(1: 1,000$; catalog no. 8480); Met (1:1,000; catalog no. 8198), CD44 (1:1,000; catalog no. 3570), T cell factor 1 (TCF1; 1:1,000; catalog no. 2203), lymphoid enhancer binding factor 1
(LEF1; 1:1,000; catalog no. 2230), cyclin D1 (1:1,000; catalog no. 2978), MMP7 (1:1,000; catalog no. 3801), c-Myc $(1: 1,000$; catalog no. 5605), $\beta$-actin $(1: 1,000$; catalog no. 4970; Cell Signaling Technology, Inc.), N-cadherin (1:500; catalog no. WL01047; Wanleibio Co., Ltd.), Ki-67 (1:500; catalog no. WL01384a; Wanleibio Co., Ltd.), VEGF-C (1:400; catalog no. BA0548; Wuhan Boster Biological Technology, Ltd.) and Lamin B1 (1:1,000; catalog no. 66095-1-Ig; Proteintech Group, Inc.). Subsequent to washing the membranes with TBST, the membranes were incubated with the anti-rabbit and anti-mouse secondary antibodies conjugated to horseradish peroxidase $(1: 1,000$; catalog nos. 7076 and 7074; Cell Signaling Technology, Inc.) for $1 \mathrm{~h}$ at room temperature. After washing the membranes with TBST, the membranes were ameliorated using an ECL kit (Vazyme Biotech Co., Ltd.), followed by obtaining images of the emitted signals using a ChemisScope-4300 imager (Clinx Science Instruments Co., Ltd.).

Cell viability assay. Cell growth rate was assessed by the CCK-8 Cell Counting kit (cat. no. A311-01/01; Vazyme Biotech Co., Ltd.) according to the manufacturer's instructions. After $48 \mathrm{~h}$ of transfection, the untreated control, negative control vector, scramble shRNA-transfected-SREBP1 and SREBP1-expression vector-transfected cells were seeded in a 96-well plate and subsequently followed by 24,48 and $72 \mathrm{~h}$ incubation at $37^{\circ} \mathrm{C}$. At every time point, $10 \mu \mathrm{l}$ CCK- 8 solution was added to each well of the plates and the cells were incubated for another $1 \mathrm{~h}$ at $37^{\circ} \mathrm{C}$ in darkness. The absorbance value at $450 \mathrm{~nm}$ was detected to represent cell viability using a microplate reader (BioTek Instruments, Inc.). Assays were repeated at least three times.

Wound healing assay. For the wound healing assay, ECA-109-NC, ECA-109-shSREBP1, KYSE-150-Vector and KYSE-150-SREBP1 cells were seeded onto 24-well plates to form a cell monolayer ( $80 \%$ confluence). The cell layer was mechanically disrupted using a sterile $200-\mu$ l pipette tip to generate a linear wound, washed three times with PBS to remove floating cells or debris and then cultured with serum-free medium (RPMI-1640; Thermo Fisher Scientific, Inc.). Images were captured $24 \mathrm{~h}$ after scraping using a light microscope (Olympus Corporation) (magnification, x200). The wound area for each condition was quantified using Image-Pro Plus version 6.0 software (Media Cybernetics, Inc.) and the migration rate was calculated using GraphPad Prism 5.0 software (GraphPad Software, Inc.) at each time point in three independent samples.

Cell invasion assay. Cell invasion was analyzed using 24-well Transwell plates with a pore size of $8 \mu \mathrm{m}$ (Corning Life Sciences). For invasion assay, the Transwell inserts were pre-coated with $25 \mu \mathrm{l}$ Matrigel (BD Biosciences) at $37^{\circ} \mathrm{C}$ and the plates were incubated for $24 \mathrm{~h}$ at $37^{\circ} \mathrm{C}$ and then ECA-109-NC, ECA-109-shSREBP1, KYSE-150-Vector and KYSE-150-SREBP1 cells $\left(2 \times 10^{5}\right)$ were suspended in $200 \mu 1$ serum-free RPMI-1640 medium and seeded on the upper chamber. The lower chamber was filled with $600 \mu \mathrm{l}$ RPMI-1640 containing 10\% FBS as an attractant. After incubation for $24 \mathrm{~h}$, the non-migrated cells in the upper chamber 
were removed using a cotton swab. Invaded cells were fixed with $4 \%$ cold paraformaldehyde and stained with $0.2 \%$ crystal violet (CoWin Biosciences) at room temperature for $15 \mathrm{~min}$. The stained cells were counted at $\times 200$ magnification under an light inverted microscope (Olympus Corporation). At least three different experiments were performed and data are presented as the mean number of stained cells from five randomly chosen fields.

ELISA assay. The expression levels of MMP9, VEGF-A and VEGF-C in culture medium were determined using quantitative ELISA kits (cat. nos. EK1M09-96, BMS277-2, EK1154-96; Hangzhou Multisciences Biotech, Co., Ltd.) according to the manufacturer's instructions.

Immunocytochemistry. Immunofluorescence was used to detect the location and expression of target proteins (E-cadherin, Vimentin) as described previously $(7,39)$. Cells were grown on glass coverslips and fixed in $4 \%$ paraformaldehyde at $4{ }^{\circ} \mathrm{C}$ for $30 \mathrm{~min}$, rinsed three times with PBS, permeabilized with $0.5 \%$ Triton X-100 for $10 \mathrm{~min}$ and blocked at room temperature for 30 min using PBS containing 5\% BSA. The cells on the coverslips were incubated with the indicated primary antibodies against: E-cadherin (1:200; catalog no. 14472; Cell Signaling Technology, Inc.), Vimentin (1:200; catalog no. 5741; Cell Signaling Technology, Inc.) and Ki-67 (1:100; catalog no. 27309-1-AP; ProteinTech Group, Inc.) at $4^{\circ} \mathrm{C}$ overnight, followed by incubation at room temperature for $1 \mathrm{~h}$ with Cy3 or FITC-conjugated secondary antibodies $(1: 1,000$; cat. nos. ab6717, ab6939 and ab97035; Abcam). The nuclei were counterstained using DAPI (Sigma-Aldrich; Merck KGaA) at room temperature in the dark for $10 \mathrm{~min}$. Images were captured using a fluorescence microscope (BX51; Olympus Corporation; magnification, $\mathrm{x} 200)$ and analyzed using ImageJ version 1.46 software (National Institutes of Health).

Animal experiments. A total of 20 female BALB/c-nu mice (age, 6 weeks; weight, 18-20 g) were purchased from Cavens Laboratory Animal Inc. (http://www.cavens.com.cn/). After 5 days of acclimatization in the Laboratory Animal Research Center of Jiangsu University at a constant temperature at $25^{\circ} \mathrm{C}$ and humidity from $50-70 \%$ on a $12 / 12 \mathrm{~h}$ light/dark cycle with free access to food and water, the mice were subcutaneously injected in the right thigh with $7 \times 10^{6}$ ECA-109-sh-SREBP1, ECA-109-NC or ECA-109 cells suspended in $100 \mu \mathrm{l}$ PBS and injected subcutaneously into the right dorsal flanks of mice, while mice only subcutaneously injected with $100 \mu 1$ PBS were treated as the control group. All mice were randomized assigned into 4 groups $(n=5)$ and the investigators were blinded to the group assignment. The mean fold-change in tumor size was plotted for each animal group. The tumor volume was measured every 4 days using a Vernier caliper and calculated as follows: Tumor size $=(\pi \mathrm{x}$ length $\mathrm{x}$ width $\mathrm{x}$ height) $/ 6$. Tumor tissues were harvested for molecular analysis and the standard deviation values were determined for the normalized values of each sample. For lung metastasis formation, ten mice were randomly selected and divided equally into two groups. The mice were injected via the lateral tail vein with $1 \times 10^{6}$ ECA-109-NC cells or ECA-109-shSREBP1 cells suspended in $100 \mu \mathrm{l}$ PBS. Tumor sizes were measured every 4 days, and mice were sacrificed on day 28 . The mice with a weight loss $>25 \%$ of total body weight and a maximum tumor size of $428 \mathrm{~mm}^{3}$ were defined as humane endpoints. The mice were euthanized 4 weeks after injection by $\mathrm{CO}_{2}$ inhalation gradually increasing in concentration in sealed chamber $(45 \times 35 \times 30 \mathrm{~cm})$ with flow meter $(3-5 \mathrm{~min}$; with the flow rate of $2.5 \mathrm{l} / \mathrm{min}$ and the volume displacement rate of $28 \% / \mathrm{min}$ ), and death was confirmed by ceased breathing, cardiac arrest and pallid eyes. The lungs were removed, (10\%, neutrally buffered, stored at $4^{\circ} \mathrm{C}$ overnight), embedded in paraffin, deparaffinized sections $\left(5-\mu \mathrm{m}\right.$ thick) and incubated at $60^{\circ} \mathrm{C}$ for $2 \mathrm{~h}$ followed by deparaffinization with xylene and rehydration in concentrations of $100,95,85$ and $75 \%$ alcohol, respectively. Subsequently, the sections were stained with hematoxylin and eosin (H\&E). The metastatic foci in the lungs were counted manually under a light microscope (magnification, x100; BX51; Olympus Corporation). All animal experiments were performed in strict accordance with the principles and procedures approved by The Animal Experimentation Ethics Committee of Jiangsu University (approval number, UJS-IACUC-AP-2020032559).

Statistical analyses. All in vitro experiments were repeated at least three times. The data were analyzed using GraphPad Prism 5.0 software (GraphPad Software, Inc.), and the values are presented as the mean \pm standard deviation. Differences between two groups were analyzed using an unpaired Student's t-test or using a paired Student's t-test when comparing the SREBP1 expression between tumor and non-tumor tissues from the same patient. One-way ANOVA with Tukey's post hoc test were used for multiple group comparisons. The association between SREBP1 and clinicopathological features was assessed using $\chi^{2}$ tests. $\mathrm{P}<0.05$ was considered to indicate a statistically significant difference.

\section{Results}

SREBP1 expression is elevated in ESCC tissues and cell lines. Expression levels of SREBP1 were investigated through bioinformatic analysis using Oncomine to determine whether SREBP1 is aberrantly expressed in ESCC. Results demonstrated that SREBP1 mRNA expression levels in ESCC tumors were significantly higher compared with normal esophageal tissues in two independent datasets (Fig. 1A) (37,38). Similarly, data from the IHC staining showed consistently higher levels of SREBP1 in primary ESCC tissues (32/77, 41.6\%) compared with normal non-neoplastic tissues $(5 / 77,6.5 \%)$. As presented in the Fig. 1B, SREBP1 was primarily located in the cytoplasm of ESCC or normal cells. The association between SREBP1 expression levels and clinicopathological features was further analyzed. IHC of human ESCC samples revealed that SREBP1 expression was significantly associated with tumor differentiation, lymphatic metastasis and Ki-67 expression (Table I). In addition, the expression levels of SREBP were significantly higher in ESCC tumors compared with adjacent normal tissues, as detected using western blotting and RT-qPCR ( $<<0.001$; Fig. 1C). The expression levels of SREBP1 and mature (m)SREBP1 were increased in ESCC tissues compared with the matched normal tissues, and the difference in SREBP2 expression was not significant (Figs. 1D and S1). SREBP1 expression levels in ESCC cell 
A
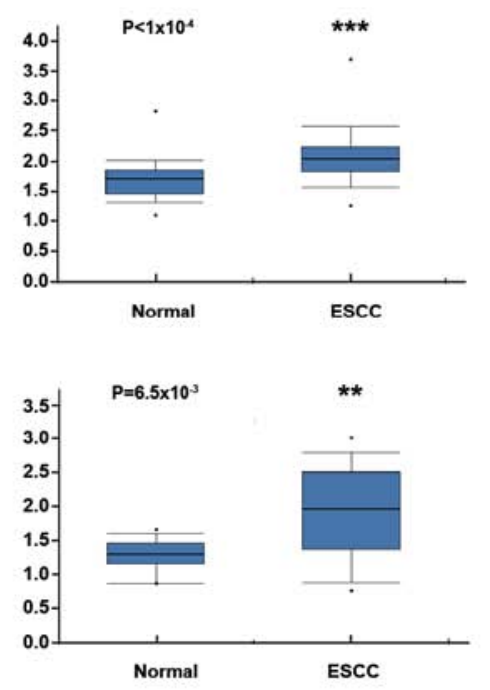

C

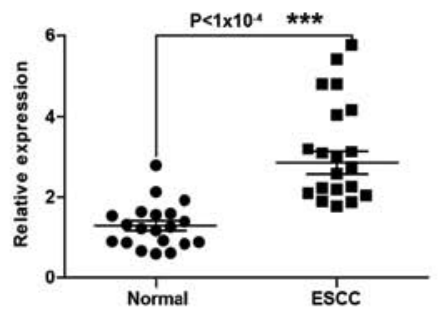

E

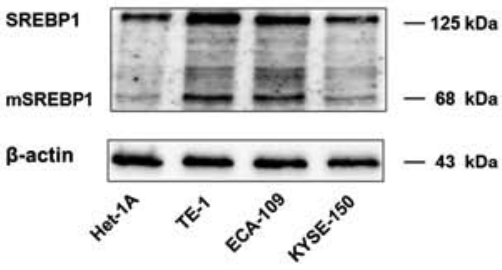

B
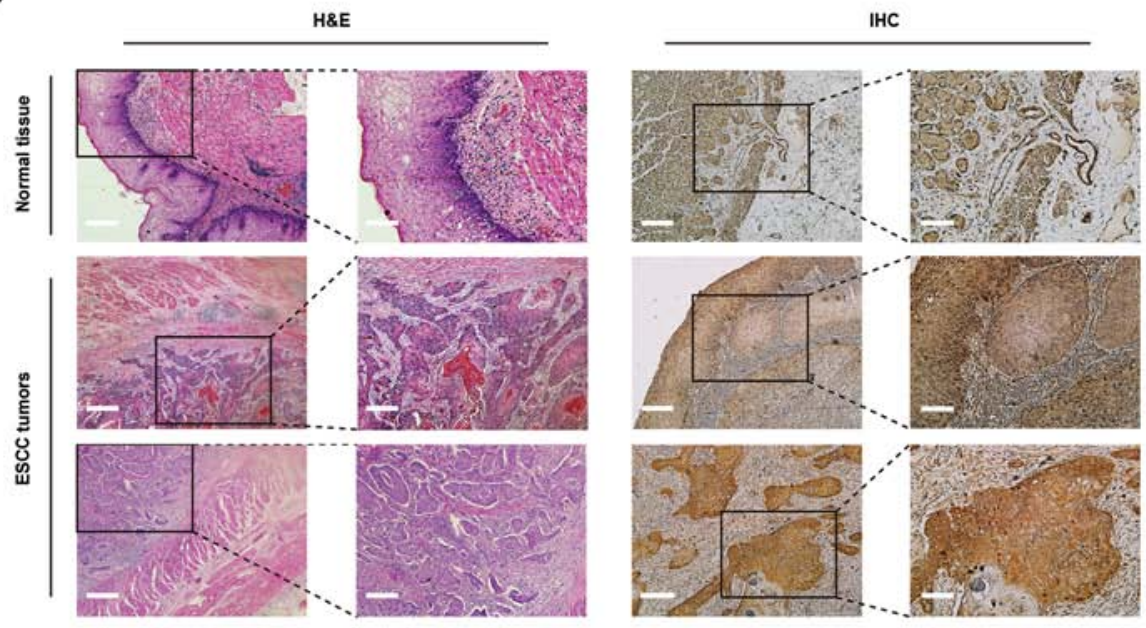

D

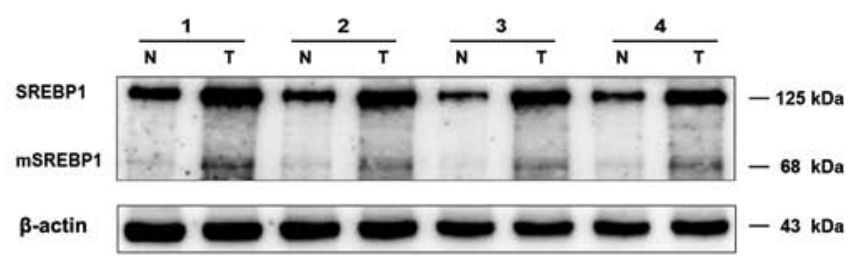

F
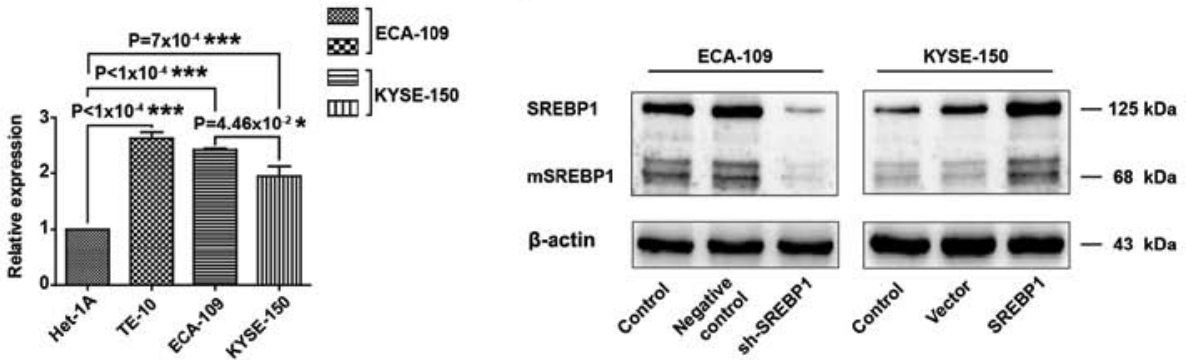

Figure 1. Enhanced SREBP1 expression levels in ESCC tumors and cells. (A) Gene expression analysis using the Oncomine database showed mRNA levels of SREBP1 in two datasets comparing normal esophageal tissue with ESCC tumors. Normal, $\mathrm{n}=56$ and cancer, $\mathrm{n}=56$ (top graph); normal, $\mathrm{n}=17$; cancer, $\mathrm{n}=17$ (bottom graph). ${ }^{* *} \mathrm{P}<0.01,{ }^{* * *} \mathrm{P}<0.001$ vs. Normal. (B) H\&E staining showing the cellular architecture and representative IHC images showing SREBP1 expression levels in ESCC tumors and normal tissues. Left panel scale bars, $100 \mu \mathrm{m}$. Right panels are magnifications of the area marked by lines (scale bars, $50 \mu \mathrm{m}$ ). (C) Relative mRNA levels of SREBP1 were determined using reverse transcription-quantitative PCR in 20 paired ESCC tumors and adjacent normal tissue samples. Results were normalized to the expression level of $\beta$-actin in each sample. Data analyzed using a paired Student's t-test. ${ }^{* * * *} \mathrm{P}<0.001$. (D) Representative expression levels of SREBP1 were detected in four matched tumors and adjacent normal tissues using western blotting. (E) Relative protein expression levels of SREBP1 in three different ESCC cell lines and one immortalized esophageal epithelial cell line were analyzed using western blotting ${ }^{* * * *} \mathrm{P}<0.001$. (F) The effects of SREBP1-knockdown or overexpression were analyzed using western blotting. mSREBP1, mature sterol regulatory element-binding protein 1; SREBP1, sterol regulatory element-binding protein 1; ESCC, esophageal squamous cell carcinoma; H\&E, hematoxylin and eosin; IHC, immunohistochemistry; N, normal tissue; T, tumor.

lines were measured to investigate the potential effect of SREBP1 in ESCC. The results demonstrated that SREBP1 protein expression was higher in all three ESCC cell lines (TE-1, ECA-109 and KYSE-150) compared with the normal immortalized cell line Het-1A (Fig. 1E). Quantitative protein analysis revealed that the relative expression of SREBP1 protein in TE-1, ECA-109, and KYSE-150 cells was 2.62, 2.41 , and 1.95 times that of Het-1A cell, respectively $(\mathrm{P}<0.05$; Fig. 1E). Notably, the ECA-109 and TE-1 cell lines had higher levels of SREBP1 expression, whereas KYSE-150 cells had relatively low expression. SREBP1 was then knocked-down in ECA-109 cells and overexpressed in KYSE-150 cells to functionally validate the role of SREBP1 in ESCC. Compared with the control and negative control groups, the relative expression level of SREBP1 was significantly decreased in the shRNA-transfected ECA-109 cells, and SREBP1 expression level was increased in the plasmid-treated KYSE-150 cells (Fig. 1F). According to the results presented in Fig. S2, the most effective shRNA (sh1), Bank Id NM_004176, was selected for the follow-up experiments. Collectively, these results demonstrated that SREBP1 is highly expressed in ESCC tumors and cells. 
A

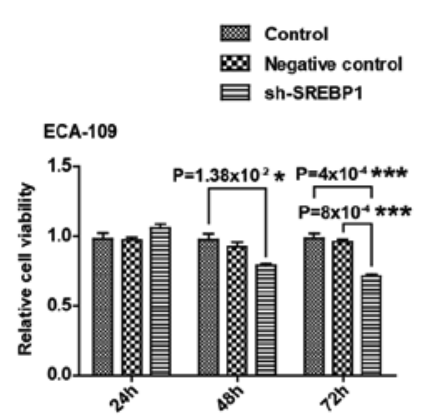

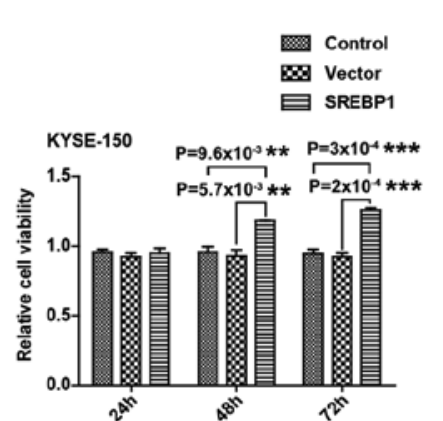

C

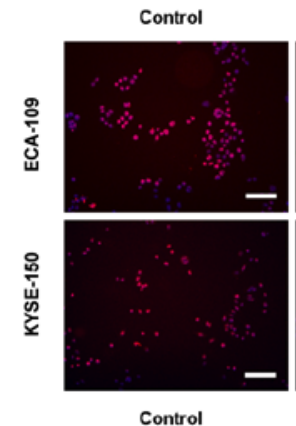

E
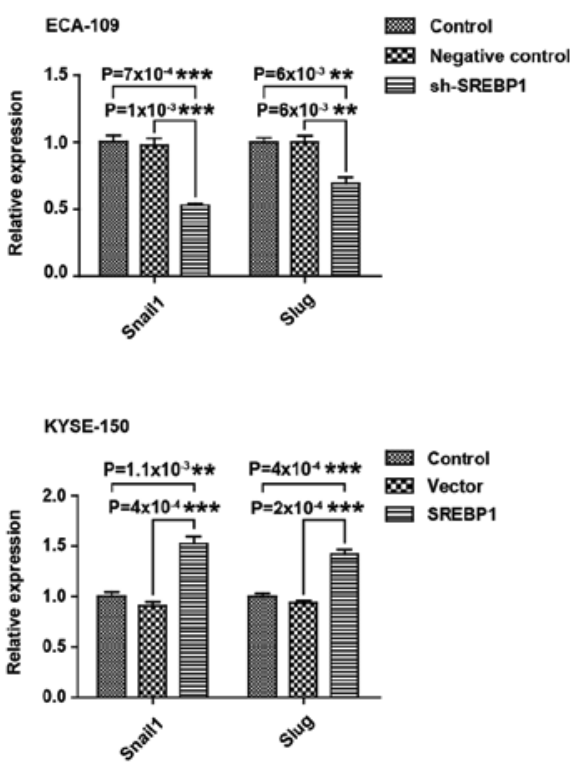

SREBP1

$\mathbf{F}$
B

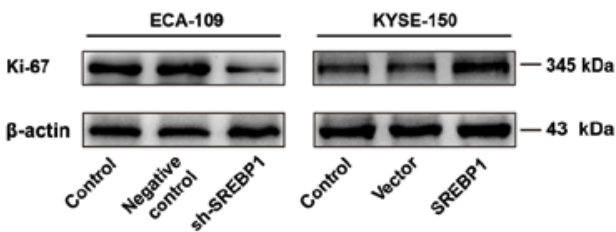

D

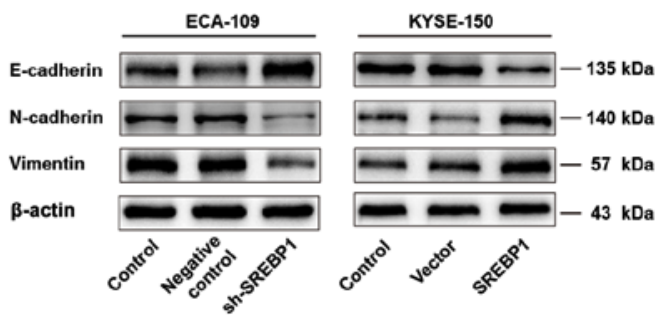

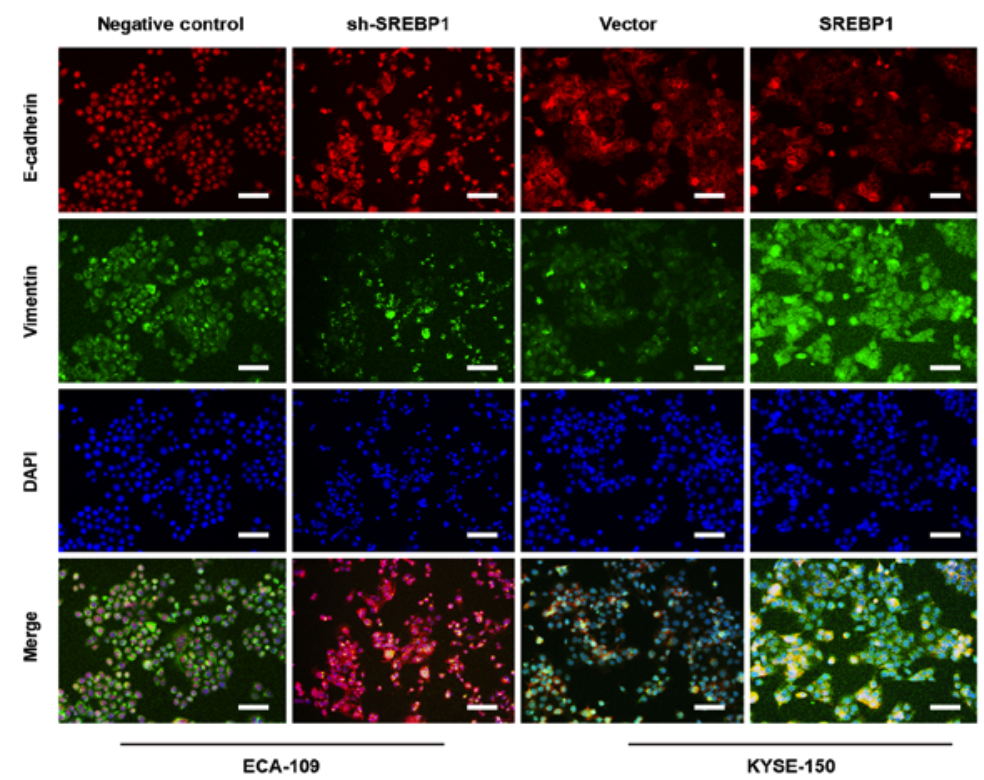

Figure 2. Effects of SREBP1 on proliferation and epithelial-mesenchymal transition process. (A) After the silencing of SREBP1 by shRNA or transfection of the SREBP1 expression vector in ECA-109 or KYSE-150 cells for 24,48 and $72 \mathrm{~h}$, cell viability was examined using a CCK- 8 assay. Data were analyzed using one-way ANOVA and Tukey's post hoc test. Expression levels of Ki-67 were analyzed in SREBP1 shRNA-expressing ECA-109 cells or SREBP1-overexpressing KYSE-150 cells at $48 \mathrm{~h}$ using (B) western blotting and (C) immunofluorescence staining (scale bars, $50 \mu \mathrm{m}$ ). (D) Representative blots showing the protein expression level changes of E-cadherin, N-cadherin and Vimentin from SREBP1-knockdown and related control ECA-109 cells, and SREBP1-overexpressing and related control KYSE-150 cells. $\beta$-actin served as the internal control. (E) Expression levels of Snail1 and Slug in ECA-109 and KYSE-150 cells treated with control, negative control of sh-SREBP1 for $48 \mathrm{~h}$ analyzed using reverse transcription-quantitative PCR. Data were analyzed using one-way ANOVA and Tukey's post hoc test. (F) Immunofluorescence staining showing the expression levels of E-cadherin (red), Vimentin (green) and DAPI in ECA-109 and KYSE-150 cells with different expression levels of SREBP1. Magnification, $x 200$. Scale bars, $50 \mu \mathrm{m} .{ }^{*} \mathrm{P}<0.05,{ }^{* * *} \mathrm{P}<0.01,{ }^{* * * *} \mathrm{P}<0.001$. SREBP1, sterol regulatory element-binding protein $1 ;$ sh, small hairpin.

Knockdown of SREBP1 reduces cellular proliferation and inhibits EMT. The proliferation changes in ECA-109 and KYSE-150 cells were detected using a CCK-8 assay, and $\mathrm{Ki}-67$ expression levels were investigated to explore the role of SREBP1 in the progression of ESCC cells. Results demonstrated that knockdown of SREBP1 significantly inhibited the cell viability of ECA-109 cells, although this was not as notable at 2 days after seeding compared with 3 days after seeding (Fig. 2A). Furthermore, this suppressive effect of proliferation was further confirmed by analysis of Ki-67 expression (Fig. 2B and C). SREBP1 ectopic overexpression effectively increased the cell viability and proliferation of KYSE-150 cells compared with the control group $(\mathrm{P}<0.05$; Fig. 2A-C). 
Whether SREBP1 affects the EMT process in ESCC was also investigated. Notably, SREBP1-overexpressing KYSE-150 cells became scattered and adopted the typical spindle-like morphology of mesenchymal cells (Fig. S3). The expression levels of multiple EMT-related factors post-SREBP1-knockdown or overexpression were analyzed using western blotting. In agreement with the phenotypic changes associated with SREBP1-overexpression, the expression levels of mesenchymal markers, including $\mathrm{N}$-cadherin and Vimentin, were increased and the expression levels of the epithelial marker E-cadherin were decreased. Opposite results were observed on SREBP1-knockdown (Fig. 2D). The expression patterns of two other typical EMT-associated transcription factors, Snaill and Slug, were also significantly reduced in SREBP1-knockdown cells and significantly increased in SREBP1-overexpressing KYSE-150 cells $(\mathrm{P}<0.01$; Fig. $2 \mathrm{E})$. The colocalization of E-cadherin and Vimentin, compared to each other under the same views was assessed using immunofluorescence analysis as described previously (7,40). Following SREBP1-knockdown and overexpression to further confirm that EMT is regulated by SREBP1 ECA-109 cells with SREBP1-knockdown exhibited increased E-cadherin expression levels compared with the negative control group, whereas the upregulation of SREBP1 in KYSE-150 cells enhanced Vimentin expression levels compared with the control group (Fig. 2F). These results suggested that SREBP1 promoted proliferation and induced EMT in ESCC cells.

Knockdown of SREBPI suppresses cellular mobility and proliferation via Wnt/ $\beta$-catenin signaling. Gene expression levels in ESCC cells were analyzed using RT-qPCR to elucidate the mechanisms by which SREBP1 modulates metastasis. As presented in Fig. 3A, the expression levels of the vascularization-related proteins VEGF-A and VEGF-C and the invasion and metastasis-related proteins MMP9, but not MMP2, were significantly enhanced after SREBP1-overexpression in KYSE-150 cells, but significantly downregulated after SREBP1-knockdown in ECA-109 cells ( $\mathrm{P}<0.05$; Fig. 3A). Furthermore, these expression levels were detected using ELISA, and the results revealed that the expression levels of MMP9, VEGF-A and VEGF-C were significantly suppressed in SREBP1-knockdown ECA-109 cells, but significantly upregulated in SREBP1-overexpressing KYSE-150 cells $(\mathrm{P}<0.001$; Fig. 3B). Wound healing and Transwell assays were then performed to ascertain whether SREBP1 affected the migration and invasion abilities of ESCC cells. The wound healing assay revealed that ECA-109 cells with SREBP1-knockdown demonstrated significantly slower closure of the wound area compared with the control cells. Conversely, SREBP1-overexpression increased the migration of KYSE-150 cells $(\mathrm{P}<0.001$; Fig. 3C). Moreover, the Transwell invasion assay revealed that silencing SREBP1 significantly reduced the number of cells on the membrane filters compared with those in the control, whereas overexpressing SREBP1 significantly increased the number of invaded cells ( $\mathrm{P}<0.01$; Fig. 3D). Collectively, these results suggested that SREBP1 contributes to the migration and invasion capacity of ESCC cells.

SREBP1 is associated with the regulation of fatty acid synthesis in response to inhibition of $\beta$-catenin (30). Thus, SREBP1 may be involved in cancer cell proliferation and metastasis via the $\mathrm{Wnt} / \beta$-catenin signaling pathway. The activity of TCF/LEF in Wnt signaling was analyzed to confirm this hypothesis. TCF1 and LEF1 were markedly decreased in SREBP1-knockdown ECA-109 cells compared with the negative control, suggesting that knockdown of SREBP1 inhibited TCF/LEF activity. By contrast, overexpression of SREBP1 markedly elevated TCF1 and LEF1 levels in KYSE-150 cells (Fig. 3E). The expression levels of GSK-3 $\beta$ and $\beta$-catenin were further investigated to evaluate the association between SREBP1 and Wnt/ $\beta$-catenin signaling. As illustrated in Fig. 3E, the expression level of $\mathrm{p}-\mathrm{GSK}-3 \beta$ was reduced by the knockdown of SREBP1 compared with the control. The expression levels of $\beta$-catenin were also markedly decreased in ECA-109 SREBP1-depleted cells compared with the control. The observations in ECA-109 cells demonstrated that knockdown of SREBP1 notably decreased the expression levels of p-GSK-3 $\beta$, whereas expression levels of total GSK-3 $\beta$ were not changed (Fig. 3E). Furthermore, the expression levels of Wnt pathway downstream targets, including c-Myc, c-Jun, Met, MMP7, CD44 and cyclin D1, were also examined. Downregulating SREBP1 in ECA-109 cells reduced the expression levels of cyclin D1 and CD44 (Fig. 3E) but had no observable effect on c-Myc, c-Jun, MMP7 and Met levels (Fig. S4). Conversely, SREBP1-overexpression in KYSE-150 cells increased the expression levels of CD44 and cyclin D1. Moreover, the aforementioned hypothesis was further confirmed in ECA-109 cells treated with the Wnt/ $\beta$-catenin pathway-specific antagonist ICG-001. In ECA-109 cells, ICG-001 markedly inhibited the Wnt/ $\beta$-catenin pathway, as indicated by the reduced expression levels of nuclear $\beta$-catenin, Snail, cyclin D1 and Ki-67. As expected, the expression levels of these proteins were further decreased after adding ICG-001 to the SREBP1 interference group (Fig. 3F). Furthermore, ICG-001 markedly inhibited the viability of KYSE-150 cells overexpressing SREBP1 in a dose-dependent manner (Fig. 3G). Overall, these observations indicated that the $\mathrm{Wnt} / \beta$-catenin signaling pathway serves a functional role in SREBP1-induced proliferation, migration and invasion in ESCC.

SREBPI activates the Wnt/ $\beta$-catenin pathway primarily through SCD1. The expression levels of SCD1, which is the target gene of SREBP1 and known regulatory factor of the $W n t / \beta$-catenin pathway, were examined to further elucidate the regulatory effect of SREBP1 on Wnt/ $\beta$-catenin signaling $(31,32)$. As presented in Fig. 4A, SCD1 was elevated in KYSE-150 cells overexpressing SREBP1, and SREBP1-knockdown resulted in a lower expression level of SCD1 in ECA-109 cells. The effect of SCD1 siRNA on the activation of $\mathrm{Wnt} / \beta$-catenin induced by SREBP1 was also assessed. SCD1-knockdown markedly reduced the nuclear $\beta$-catenin expression levels regardless of whether SREBP1 was overexpressed or not, whereas there was no effect on the expression levels of SREBP1 (Fig. 4B). KYSE-150 cells were cultured in the absence or presence of Fatostatin $(10 \mu \mathrm{M})$ and Mevastatin $(10 \mu \mathrm{M})$ for $24 \mathrm{~h}$ to verify whether pharmacological inhibition of SREBP1 downregulates SCD1. Western blotting confirmed that Fatostatin, an inhibitor of SREBP that directly binds SREBP cleavage-activating protein and blocks its endoplasmic reticulum-to-Golgi transport (45), reduced the expression levels of mSREBP1 and SCD1 in the nucleus but not in the cytoplasm, and blocked the nuclear translocation 
A
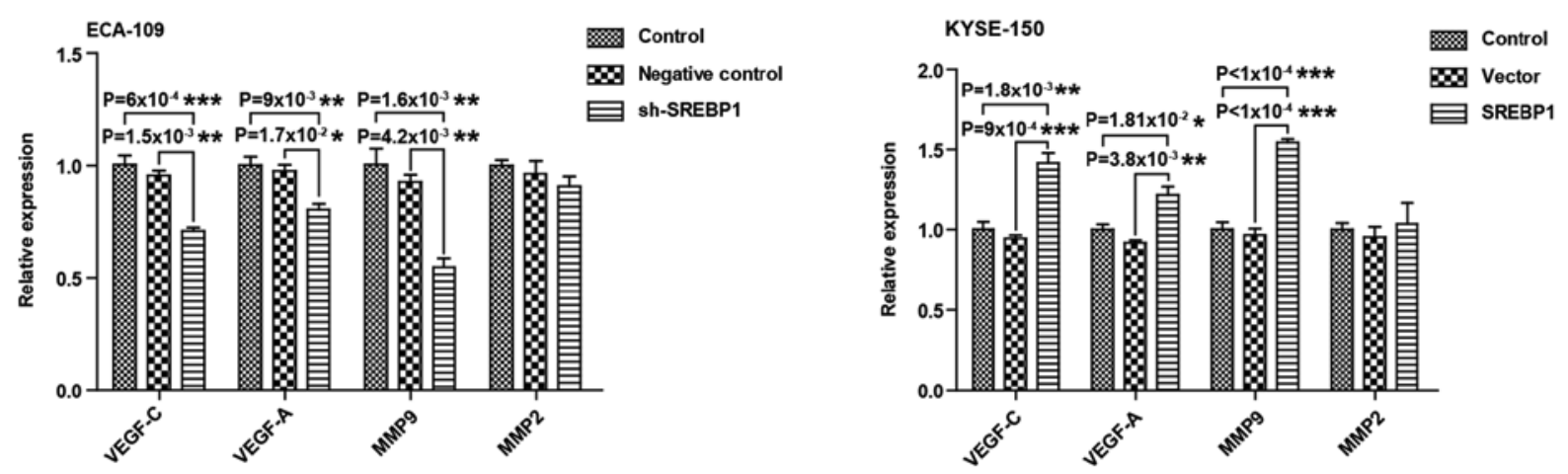

B
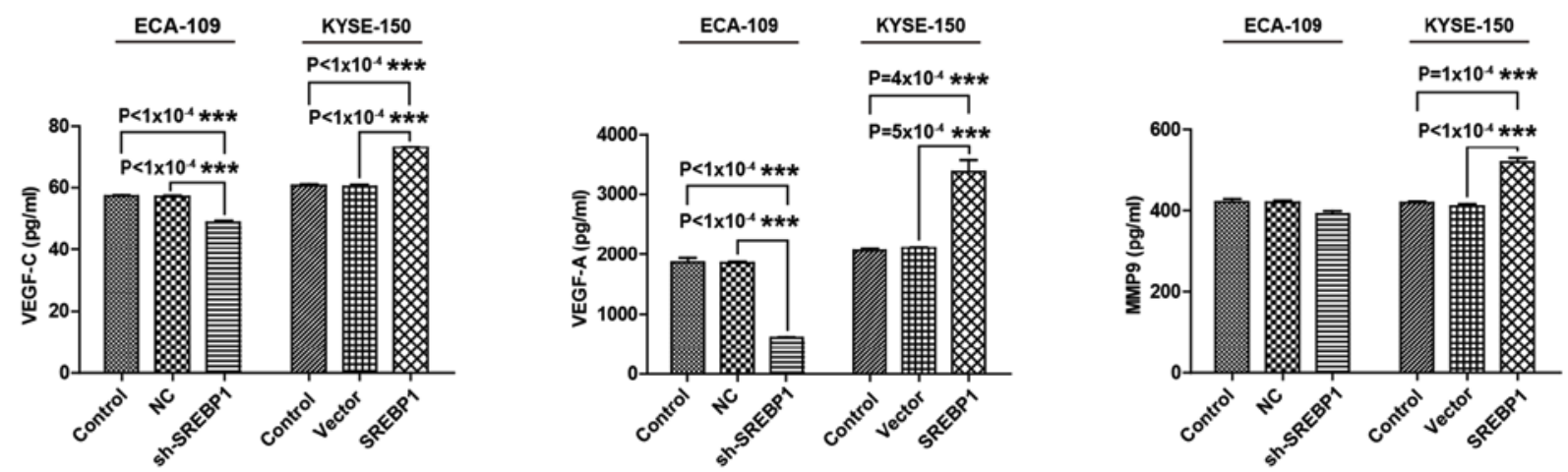

C
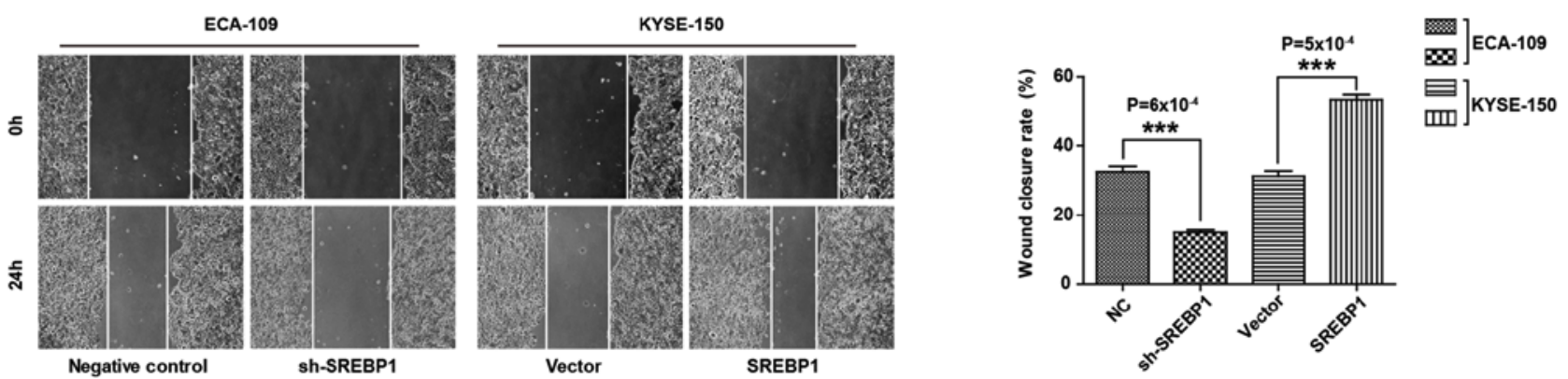

D
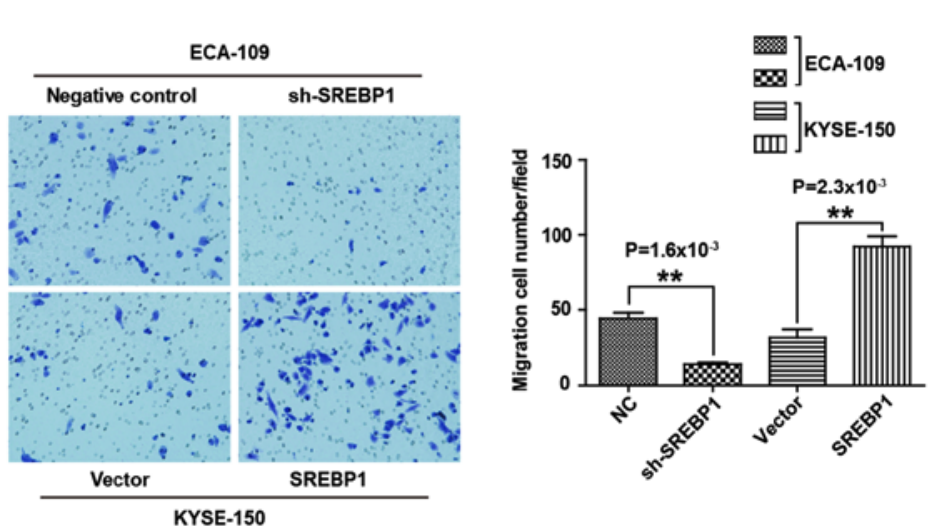

$\mathbf{E}$

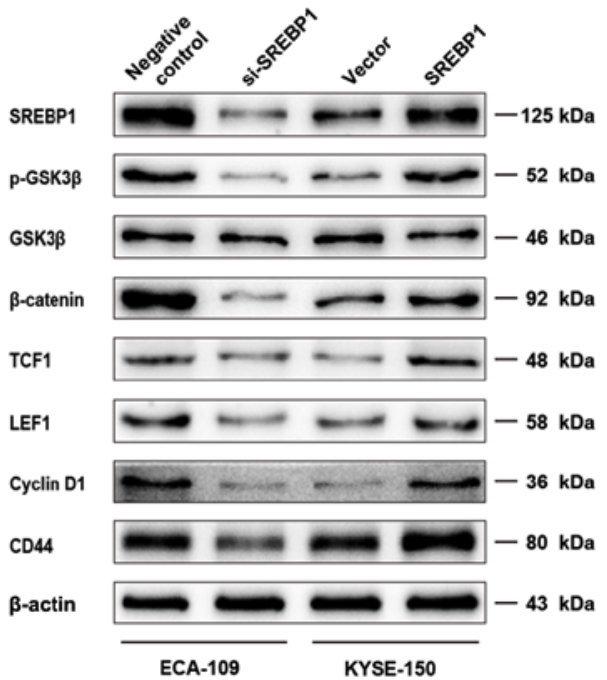

Figure 3. Effects of SREBP1 on ESCC proliferation and metastasis are mediated by activating the Wnt/ $\beta$-catenin signaling pathway. (A) mRNA and (B) protein expression levels of MMPs and VEGFs, including MMP2, MMP9, VEGF-A and VEGF-C were examined in SREBP1-downregulated or -upregulated ESCC cells using reverse transcription-quantitative PCR and ELISA assays. Data were analyzed using one-way ANOVA and Tukey's post hoc test. The effects of SREBP1-knockdown or overexpression on the (C) migration and (D) invasion, migration of ESCC cells were assessed by wound healing (magnification, $\mathrm{x} 100$ ) and Transwell assays (magnification, x200), respectively. Data were analyzed using unpaired Student's t-tests. (E) Effects of SREBP1-depletion and SREBP1-overexpression on the Wnt/ $\beta$-catenin signaling pathway were determined using western blotting. The protein expression levels of Wnt/ $\beta$-catenin pathway target proteins cyclin D1 and CD44 were also measured. $\beta$-actin and Lamin B1 served as the loading controls. 

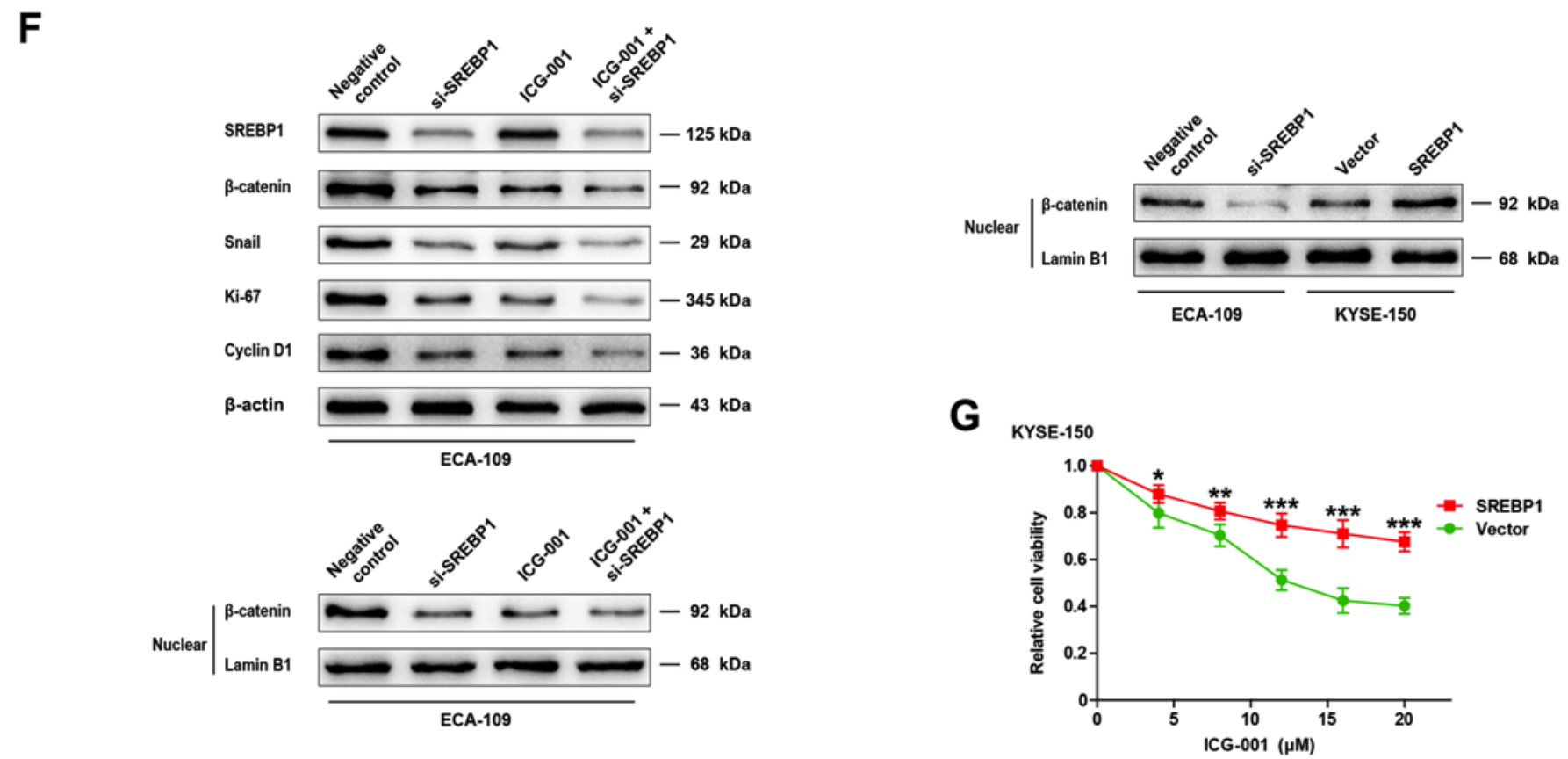

Figure 3 (Continued). (F) ECA-109-siSREBP1 cells were treated with or without $10 \mu \mathrm{M}$ ICG-001, an inhibitor of the Wnt/ $\beta$-catenin signaling pathway, and the expression levels of Wnt/ $\beta$-catenin pathway target proteins and Ki-67 were examined using western blotting. (G) A Cell Counting Kit-8 assay was used to analyze cell viability of KYSE-150 and SREBP1-overexpressing KYSE-150 cells treated with escalating doses of ICG-001. Data were analyzed using unpaired Student's t-tests. ${ }^{*} \mathrm{P}<0.05,{ }^{* *} \mathrm{P}<0.01,{ }^{* * *} \mathrm{P}<0.001$ vs. vector. SREBP1, sterol regulatory element-binding protein 1 ; ESCC, esophageal squamous cell carcinoma; sh, small hairpin; si, small interfering; p, phosphorylated; VEGF, vascular endothelial growth factor; MMP, matrix metalloproteinase; TCF1, T-cell factor 1; LEF1, lymphoid enhancer factor 1; NC, negative control.

of $\beta$-catenin (Fig. 4C). In addition, regardless of whether SREBP1was overexpressed, mevastatin that inhibits HMGCR downstream of SREBP1 decreased the expression levels of SCD1 and $\beta$-catenin in the nucleus (Fig. S5). Furthermore, the activation of the Wnt/ $\beta$-catenin pathway was measured when SREBP1 and SCD1 were inhibited by Fatostatin. As expected, Fatostatin reversed SREBP1-mediated growth promotion in a dose-dependent manner (Fig. 4D), and the expression levels of VEGF-A and MMP9 were significantly decreased (Fig. 4E) (46). Hence, these results suggested that activation of the Wnt $/ \beta$-catenin pathway by SCD1 is essential for the proliferation, EMT and metastasis of ESCC cells driven by constitutively active SREBP1.

Knockdown of SREBPI suppresses tumorigenesis and metastasis in vivo. A xenograft tumor model in nude mice was established using the SREBP1-knockdown ECA-109 cells to investigate whether SREBP1 silencing inhibits ESCC cell proliferation and metastasis in vivo. As presented in Fig. 5A and B, xenograft models inoculated subcutaneously using cells transfected with shSREBP1 had reduced abilities to form tumors compared with those in control mice, and the mean final volume of tumors transfected with shSREBP1 was 3.64-fold smaller compared with the control group 28 days after implantation. The results demonstrated that the differences in tumor growth curves were significant between the ECA-109-shSREBP1 and control groups after inoculation for 20 days $(\mathrm{P}<0.01)$, and the maximum tumor diameter $(14.84 \mathrm{~mm})$ and volume $\left(428 \mathrm{~mm}^{3}\right)$ were observed in the control group (Fig. 5C). In general, these findings demonstrated that SREBP1-knockdown suppressed proliferation in ESCC.
IHC analysis was also performed on xenograft sections. As indicated in Fig. 5C, lower SREBP1 expression levels were observed in the tumors derived from ECA-109-shSREBP1 cells, indicating that SREBP1-knockdown was maintained in vivo. Moreover, the expression levels of $\beta$-catenin, MMP9, VEGF-C and $\mathrm{Ki}-67$ were repressed in the SREBP1-knockdown xenografts compared with the ECA-109-shNC xenografts (Fig. 5D). This phenomenon suggested that SREBP1-knockdown induced a reduction in MMP9, VEGF-C and Ki-67 expression levels, promoting tumor growth and metastasis in vivo, and these data were with the aforementioned in vitro findings. Even the expression levels of SCD1 and p-GSK- $3 \beta$ were elevated in the partial region of tumors, but the overall trend showed no difference between the two groups. Furthermore, ECA-109-shSREBP1 and negative control cells were injected into the tail veins of nude mice to reveal whether SREBP1-knockdown could affect the metastatic potential of ESCC cells in vivo. The mice were sacrificed 6 weeks after injection, and the number of metastatic nodules in the lung was counted. H\&E staining demonstrated that the silencing of SREBP1 significantly decreased the number of metastatic nodules in the lung compared with that the vector group $(\mathrm{P}<0.001 ;$ Fig. $5 \mathrm{E})$. These findings suggested that SREBP1-knockdown suppressed the proliferative and metastatic properties of ESCC cells in vivo.

\section{Discussion}

SREBP1 is a critical transcription factor that controls the expression of genes important for the uptake and synthesis of lipids, such as cholesterol, fatty acids and phospholipids (47). 
A

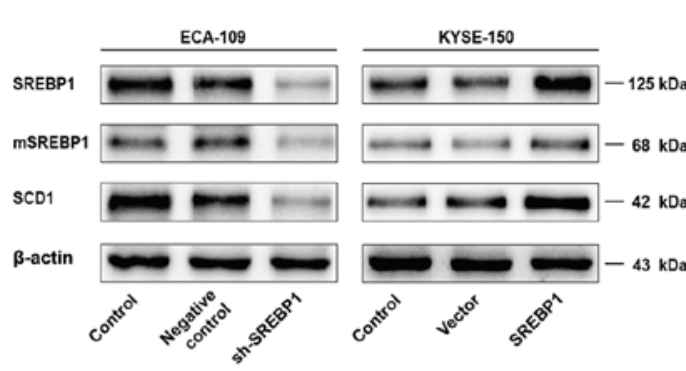

B

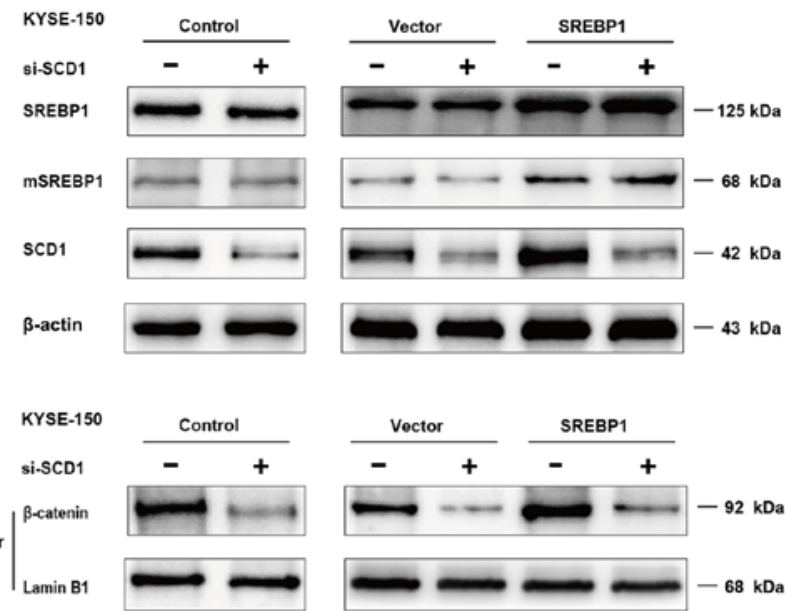

C $_{\text {KYSE-150 }}$

\begin{tabular}{|c|c|c|c|c|}
\hline Fatostatin $(\mu \mathrm{M})$ & - & - & + & + \\
\hline mSREBP1 & $\cdots$ & $\longrightarrow$ & $\longrightarrow$ & $\longrightarrow$ \\
\hline
\end{tabular}

SCD1

p.GSK33

GsKзß

$\beta$-catenin

$\beta$-actin
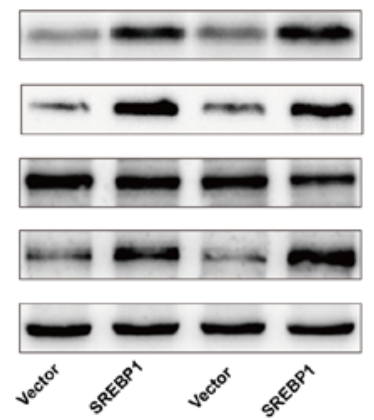

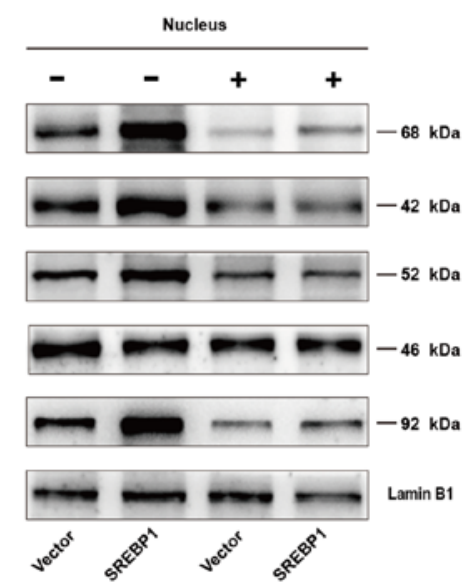

D

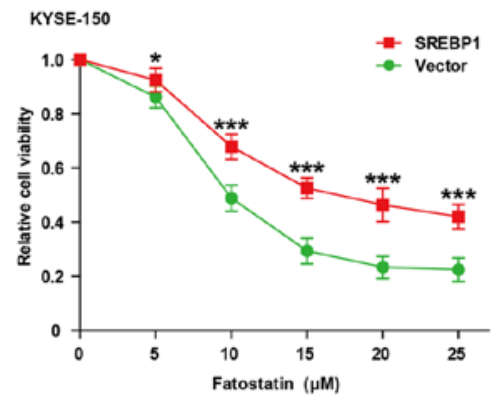

E
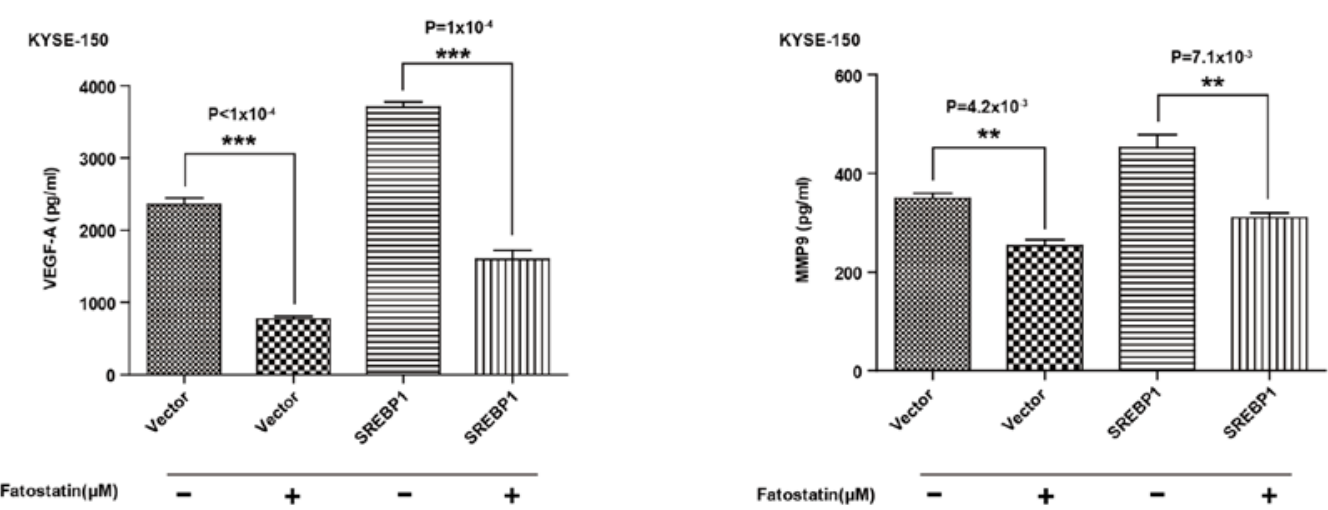

Figure 4. Silencing of SCD1 or blocking SREBP1/SCD1 desensitizes the Wnt/ק-catenin signaling in ESCC cells. (A) Expression levels of SCD1 were assessed in cell lines treated with sh-SREBP1 or SREBP1-overexprssion vector. (B) Western blotting of the proteins involved in Wnt/ $\beta$-catenin activation in KYSE-150 cells overexpressing SREBP1 or following transfection with siRNA-SCD1 or siRNA-NC for $24 \mathrm{~h}$. (C) Western blotting showing the translocations of SREBP1 and SCD1, and Wnt/ $/$-catenin pathway activation in ESCC cells treated with or without Fatostatin $(10 \mu \mathrm{M})$ for $24 \mathrm{~h}$. (D) Proliferation was assessed in cell lines treated with escalating doses of Fatostatin. Data were analyzed using unpaired Student's t-tests. ${ }^{*} \mathrm{P}<0.05,{ }^{* * * *} \mathrm{P}<0.001$ vs. vector. (E) Expression levels of VEGF-A and MMP9 were assessed in KYSE-150 cells treated with Fatostatin $(10 \mu \mathrm{M})$ for $48 \mathrm{~h}$. Data were analyzed using unpaired Student's t-tests. ${ }^{* *} \mathrm{P}<0.01$, ${ }^{* * * *} \mathrm{P}<0.001$. SREBP1, sterol regulatory element-binding protein 1; SCD1, stearoyl-CoA desaturase 1; ESCC, esophageal squamous cell carcinoma; sh, small hairpin; si, small interfering; VEGF, vascular endothelial growth factor; MMP, matrix metalloproteinase; mSREBP1, mature sterol regulatory element-binding protein 1.

Previous studies have revealed that SREBP1 is a tumor promoter and that its expression is upregulated in several malignancies, such as ovarian tumor, colon adenocarcinoma, breast cancer, etc. (24-28,48-51). Analysis using Oncomine revealed that SREBP1 expression levels were higher in ESCC tumors compared with paired normal tissues, indicating that SREBP1 may participate in carcinogenesis and tumor progression $(37,38)$. Although the multiple functions of SREBP1 have been investigated in different types of tumors, the distinct role of SREBP1 in ESCC remains unclear. Therefore, the present 

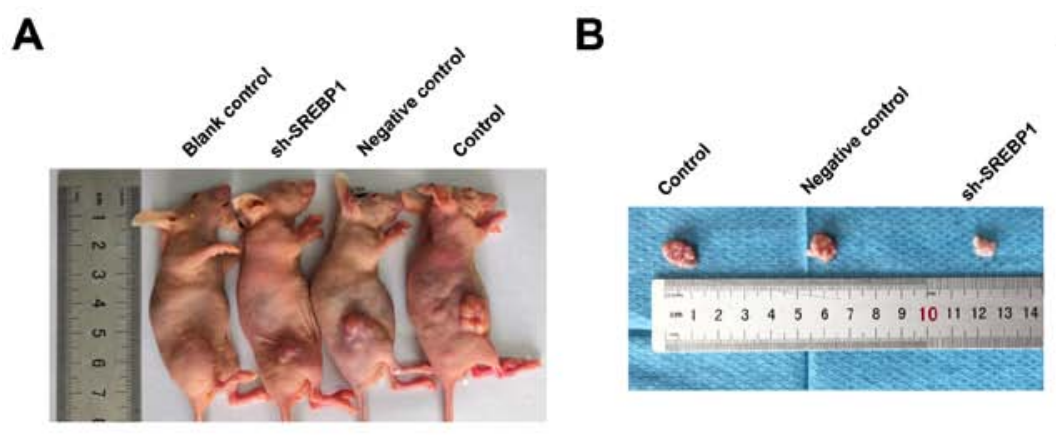

C

D

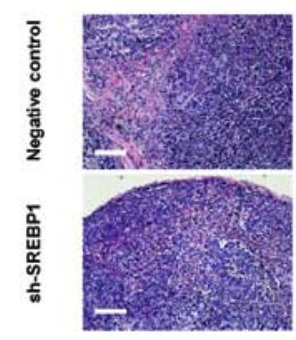

H\&E
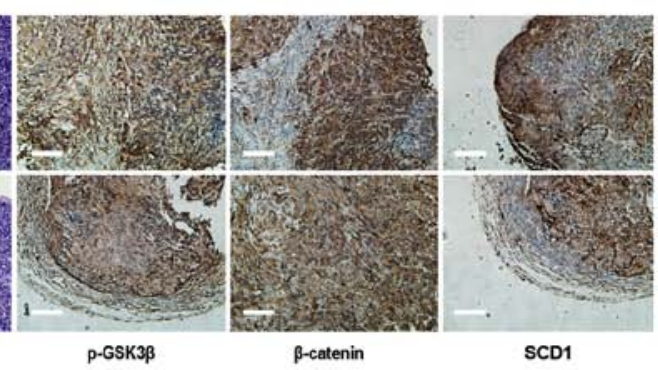

\section{I}

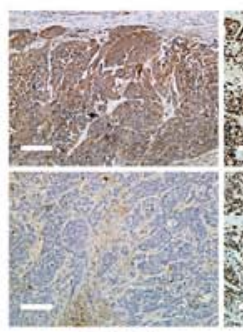

SREBP1

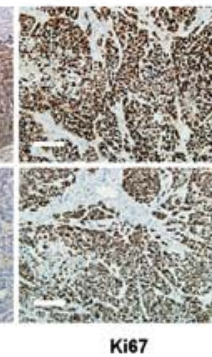

Ki67

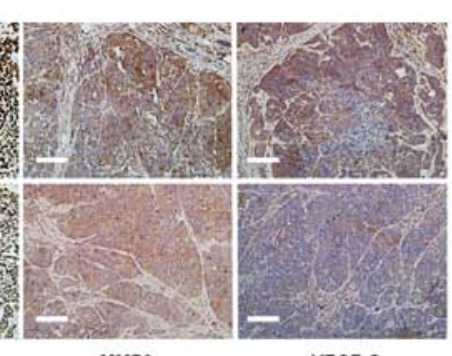

MMP9

VEGF-C

\section{E}
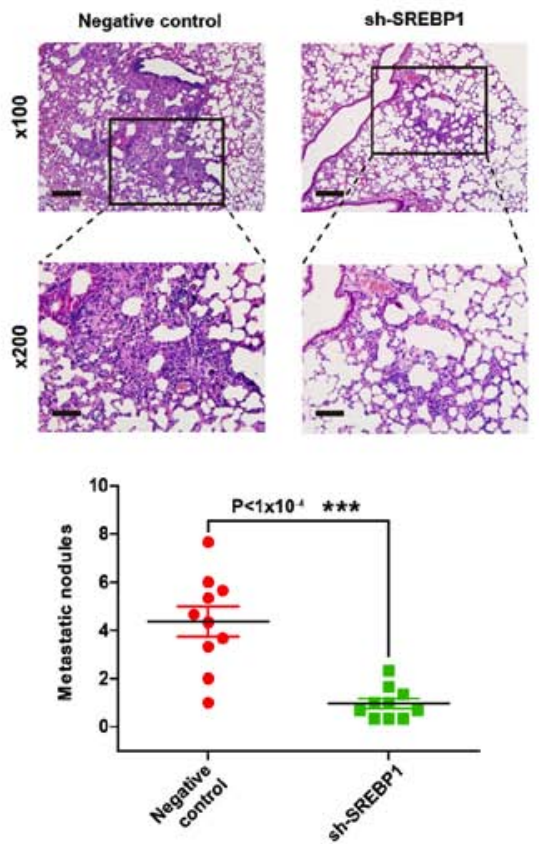

Figure 5. SREBP1-knockdown inhibits the proliferation and metastasis of ESCC cells in vivo. Images of (A) nude mice and (B) tumor xenografts with the indicated treatments. One for each control and treated groups are presented. (C) ECA-109 cells expressing control vector, negative control or sh-SREBP1 were injected subcutaneously into nude mice. $n=5 /$ group. Data were analyzed using unpaired Student's t-tests. (D) H\&E staining showing the cellular architecture, and immunohistochemistry results showed the changes of SREBP1, p-GSK-3 $\beta$, $\beta$-catenin, SCD1, Ki-67, MMP9 and VEGF-C expression levels in xenograft tumors between sh negative control and shSREBP1 groups (scale bars, $50 \mu \mathrm{m}$ ). (E) Representative images of lung metastases derived from nude mice receiving tail vein injection of ECA-109-shSREBP1 and the negative control cells are shown in H\&E staining at x100 (scale bars, $100 \mu \mathrm{m}$ ) and x200 magnification (scale bars, $50 \mu \mathrm{m})$. The number of lung metastasis is shown in the below panel. Data were analyzed using an unpaired Student's t-test. ${ }^{* *} \mathrm{P}<0.01,{ }^{* * *} \mathrm{P}<0.001$. SREBP1, sterol regulatory element-binding protein 1; ESCC, esophageal squamous cell carcinoma; sh, small hairpin; p, phosphorylated; H\&E, hematoxylin and eosin; VEGF, vascular endothelial growth factor; SCD1, stearoyl-CoA desaturase 1 ; MMP, matrix metalloproteinase; GSK-3 $\beta$, glycogen synthase kinase-3 $\beta$.

study investigated SREBP1 in patients with ESCC. IHC results demonstrated that higher expression levels of SREBP1 were detected in $41.6 \%$ of ESCC samples but in only $6.5 \%$ of the matched adjacent normal tissues. Furthermore, SREBP1 was significantly upregulated at the transcript and protein levels in ESCC tissues compared with the normal tissues. ESCC tumor tissue analysis showed that patients in the high SREBP1 expression group had significantly poorer differentiation, more lymphatic metastasis and higher Ki-67 expression levels compared with those in the low SREBP1 expression group. The present data suggested that SREBP1-overexpression may contribute to the development of ESCC, which is consistent with SREBP1-overexpression previously described in ovarian tumor $(24,48)$, glioblastoma $(25)$, prostate $(27)$, colorectal $(28)$ and breast cancer $(50,51)$, and nasopharyngeal carcinoma (NPC) (48). In addition, a number of studies have reported that SREBP1 participates in the proliferation of multiple cancer types $(24,26,35,49)$. Hence, the present study knocked-down SREBP1 in ECA-109 cells and found that SREBP1-depletion exerts a suppressive effect on proliferation, whereas overexpression of SREBP1 promoted proliferation. Unlimited proliferation leads to further malignancy of the tumor, and EMT is an essential step that occurs during cancer migration and invasion (52). Previous research has reported that SREBP1 represses E-cadherin expression levels via miRNA-18a-5p and forms corepressor complexes with Snail and HDAC1/2 in breast cancer (51). The present study showed that KYSE-150 cells overexpressing SREBP1 exhibited the characteristics of mesenchymal cells and induced higher expression levels of mesenchymal markers, including $\mathrm{N}$-cadherin and Vimentin, as well as EMT-related transcription factors, such as Snail and Slug. The changes in the expression of members of the MMP and VEGF protein families critical for tumor invasion, metastasis, angiogenesis and lymphangiogenesis were also 
analyzed (53). Results revealed that loss of SREBP1 markedly suppressed the expression levels of MMP9, VEGF-A and VEGF-C in ECA-109 cells. Additionally, the upregulation of SREBP1 in KYSE-150 cells significantly increased EMT-related transcription factors. A previous study reported that SREBP1 can suppress VEGF expression through indirect interaction with the proximal tandem Sp1 sites in human smooth muscle cells (54). VEGF-C, as a lymphangiogenic switch, is a specific factor involved in the invasiveness and metastasis of $\operatorname{ESCC}(7,11,39,55)$. Furthermore, according to the significant relationship between SREBP1 expression levels and lymphatic metastasis shown in the present study, SREBP1 was identified as a promoting factor regulating VEGF-C formation and secretion. Recently, studies have suggested that SREBP1 can participate in the metastasis of multiple tumors $(28,49,51)$. Wound healing and Transwell assays in the present study revealed that cell migration and invasion were either upregulated or downregulated in response to SREBP1-overexpression or knockdown in ESCC cells, respectively. These results indicated that SREBP1 mediated EMT and conferred metastatic potential, ultimately promoting malignancy in ESCC cells.

Recent studies have indicated that SREBP1 contributes to the regulation of cancer cell survival, proliferation and metastasis via several signaling pathways. For example, SREBP1 is involved in colorectal cancer cell invasion and metastasis via the NF- $\kappa \mathrm{B}$ signaling pathway (28). The multikinase inhibitor Sorafenib acts through the ATP-AMPK-mTOR-SREBP1 pathway to disrupt lipogenesis and trigger liver cancer cell death (56). SREBP1-mediated lipogenesis promotes proliferation and is involved in Epstein-Barr virus-driven NPC pathogenesis, which is a potential therapeutic target for locally advanced or metastatic NPC (49). In addition, previous research reported that SREBP1 mediates the repression of E-cadherin expression levels and promotes EMT and further metastasis by recruiting the Snail/HDAC1/2 repressor complex in breast cancer (51). The Wnt/ $\beta$-catenin signaling pathway is the classical signaling pathway involved in tumorigenesis and metastasis. Our previous studies showed that activation of the Wnt/ $\beta$-catenin pathway can induce EMT, allowing ESCC cells to metastasize $(7,11)$. In the present study, depletion of SREBP1 suppressed TCF1 and LEF1 activity, accumulation of $\beta$-catenin and downregulated Wnt pathway downstream target genes, including CD44 and cyclin D1; however, c-Myc, c-Jun, MMP7 and Met expression levels were not changed. By contrast, overexpression of SREBP1 increased the expression levels of p-GSK-3 $\beta$ and $\beta$-catenin in the nucleus. Furthermore, ESCC cells were treated with the specific Wnt/ $\beta$-catenin pathway inhibitor ICG-001. Results showed that ICG-001 treatment further enhanced the suppressive effects on the expression levels of Snail, cyclin D1, Ki-67 and $\beta$-catenin in SREBP1-depleted cells, whereas expression levels of SREBP1 in ESCC cells was not affected by ICG-001 treatment. Wnt/ $\beta$-catenin activity can be mediated by SCD1, especially regarding cancer stem cell-fate decisions and influencing tumor progression (17). SCD1 expression levels were significantly elevated when ESCC cells overexpressed SREBP1, and siRNA-SCD1 can markedly inhibit SREBP1-induced activation of Wnt/ $\beta$-catenin (57-60). In the present study, ESCC cells were treated with either the SREBP1 inhibitor Fatostatin or the HMGCR-specific inhibitor Mevastatin to determine whether these effects could be recapitulated by small-molecule SREBP1 inhibitors. In both cases, nuclear expression levels of SCD1 were decreased, with inhibited levels of Wnt/ $\beta$-catenin pathway-associated proteins. Thus, the synchronous inhibition of SREBP1 and SCD1 by Fatostatin caused inhibition of proliferation, EMT and metastasis. These results suggested that SREBP1 mediated ESCC cell invasion, migration and proliferation, at least in part, by activating the $\mathrm{Wnt} / \beta$-catenin pathway. Further experiments in vivo demonstrated that the knockdown of SREBP1 significantly suppressed tumor growth along with the number of metastatic nodules in the lung. Therefore, the present findings demonstrated that overexpression of SREBP1 affects the Wnt/ $\beta$-catenin pathway via SCD1, promoting proliferation and metastasis in ESCC. Based on findings that $\beta$-catenin translocated to the nucleus in tumor immune response, and that ICG-001 reversed the activation of Wnt/ $\beta$-catenin followed EMT, invasion, metastasis and radioresistance in vitro and vivo, it was hypothesized that the Wnt/ $\beta$-catenin pathway may regulate distant metastases in xenografts tumors, presumably via a coordination of SREBP1 and SCD1 $(7,41)$.

Although the present study showed that SCD1-mediated translocation of $\beta$-catenin contributed to the oncogenic activity of SREBP1 in ESCC, the function of additional genes in stimulating EMT and the pro-metastatic response induced by SREBP1 should not be ignored and further investigated in the future. Additionally, the association between SREBP1, SCD1 and the Wnt/ $\beta$-catenin signaling pathway in ESCC specimens and the potential therapeutic application of ICG-001 needs to be further clarified.

In conclusion, the present results demonstrated that the upregulation of SREBP1 in ESCC tumors and cells was significantly associated with proliferation and metastasis. In addition, SREBP1 served a critical role in inducing the proliferation, migration and invasion of human ESCC cells, inducing EMT and the upregulation of VEGFs and MMPs. Furthermore, SREBP1-depletion resulted in the suppression of SCD1 and activity of the $\mathrm{Wnt} / \beta$-catenin signaling pathway, thereby restraining metastasis in vivo. These findings indicate a pivotal pro-oncogenic role for SREBP1 in tumor progression and may lead to the development of novel therapeutic targets for ESCC.

\section{Acknowledgements}

Not applicable.

\section{Funding}

The present study was funded by The National Natural Science Foundation of China (grant no. 81572956), The Jiangsu Provincial Science and Technology Supporting Program (grant no. BE2017096), The Medical Innovation Team of Jiangsu Province (grant no. CXTDC2016009) and The Student Innovation Training Program Projects of Jiangsu University (grant no. 201810299262W).

\section{Availability of data and materials}

The datasets used and/or analyzed during the present study are available from the corresponding author on reasonable request. 


\section{Authors' contributions}

YZ, CM and DC conceived and designed the study. YZ and JW designed the experiments. JW and RL performed the experiments and analyzed the data. XG and YY performed the pathological analysis. YZ and DC wrote, edited and revised the manuscript. All authors have read and approved the final manuscript.

\section{Ethics approval and consent to participate}

The study was approved by The Medical Ethical Committee of the Affiliated Hospital of Jiangsu University (Zhenjiang, China), and written informed consent was obtained from all participants. All animal experiments were performed in strict accordance with the principles and procedures approved by The Animal Experimentation Ethics Committee of Jiangsu University (approval number, UJS-IACUC-AP-2020032559).

\section{Patient consent for publication}

Not applicable.

\section{Competing interests}

The authors declare that they have no competing interests.

\section{References}

1. Bray F, Ferlay J, Soerjomataram I, Siegel RL, Torre LA and Jemal A: Global cancer statistics 2018: GLOBOCAN estimates of incidence and mortality worldwide for 36 cancers in 185 countries. CA Cancer J Clin 68 (Suppl 8): S394-S424, 2018.

2. Abnet CC, Arnold M and Wei WQ: Epidemiology of esophageal squamous cell carcinoma. Gastroenterology 154: 360-373, 2018.

3. McGuire S: World cancer report 2014. Geneva, Switzerland: World Health Organization, International Agency for Research on Cancer, WHO Press, 2015. Adv Nutr 7: 418-419, 2016.

4. Wang H, Deng F, Liu Q and Ma Y: Prognostic significance of lymph node metastasis in esophageal squamous cell carcinoma. Pathol Res Pract 213: 842-847, 2017.

5. Hou H, Meng Z, Zhao X, Ding G, Sun M, Wang W and Wang Y: Survival of esophageal cancer in China: A pooled analysis on hospital-based studies from 2000 to 2018. Front Oncol 9: 548, 2019.

6. Ordóñez-Morán P and Huelsken J: Complex metastatic niches: Already a target for therapy? Curr Opin Cell Biol 31: 29-38, 2014.

7. Chen D, Li W, Liu S, Su Y, Han G, Xu C, Liu H, Zheng T, Zhou Y and Mao C: Interleukin-23 promotes the epithelial-mesenchymal transition of oesophageal carcinoma cells via the Wnt/ $\beta$-catenin pathway. Sci Rep 5: 8604, 2015.

8. Wang H, Yang X, Guo Y, Shui L, Li S, Bai Y, Liu Y, Zeng M and Xia J: HERG1 promotes esophageal squamous cell carcinoma growth and metastasis through TXNDC5 by activating the PI3K/AKT pathway. J Exp Clin Cancer Res 38: 324, 2019.

9. Zhou H, Liu Y, Zhu R, Ding F, Cao X, Lin D and Liu Z: OTUB1 promotes esophageal squamous cell carcinoma metastasis through modulating Snail stability. Oncogene 37: 3356-3368, 2018.

10. Li L, Zhou H, Zhu R and Liu Z: USP26 promotes esophageal squamous cell carcinoma metastasis through stabilizing Snail. Cancer Lett 448: 52-60, 2019.

11. Ling R, Zhou Y, Zhou L, Dai D, Wu D, Mi L, Mao C and Chen D: Lin28/microRNA-let-7a promotes metastasis under circumstances of hyperactive Wnt signaling in esophageal squamous cell carcinoma. Mol Med Rep 17: 5265-5271, 2018.

12. Pang L, Li Q, Li S, He J, Cao W, Lan J, Sun B, Zou H, Wang C, Liu R, et al: Membrane type 1-matrix metalloproteinase induces epithelial-to-mesenchymal transition in esophageal squamous cell carcinoma: Observations from clinical and in vitro analyses. Sci Rep 6: 22179, 2016.
13. Dias IH, Borah K, Amin B, Griffiths HR, Sassi K, Lizard G, Iriondo A and Martinez-Lage P: Localisation of oxysterols at the sub-cellular level and in biological fluids. J Steroid Biochem Mol Biol 193: 105426, 2019.

14. Matsudaira T, Mukai K, Noguchi T, Hasegawa J, Hatta T, Iemura SI, Natsume T, Miyamura N, Nishina H, Nakayama J, et al: Endosomal phosphatidylserine is critical for the YAP signalling pathway in proliferating cells. Nat Commun 8: 1246, 2017.

15. Ma XL, Sun YF, Wang BL, Shen MN, Zhou Y, Chen JW, Hu B, Gong ZJ, Zhang X, Cao Y, et al: Sphere-forming culture enriches liver cancer stem cells and reveals Stearoyl-CoA desaturase 1 as a potential therapeutic target. BMC Cancer 19: 760, 2019.

16. Dai S, Yan Y, Xu Z, Zeng S, Qian L, Huo L, Li X, Sun L and Gong Z: SCD1 confers temozolomide resistance to human glioma cells via the Akt/GSK3 $/ / \beta$-catenin signaling axis. Front Pharmacol 8: 960, 2018.

17. Pisanu ME, Noto A, De Vitis C, Morrone S, Scognamiglio G, Botti G, Venuta F, Diso D, Jakopin Z, Padula F, et al: Blockade of Stearoyl-CoA-desaturase 1 activity reverts resistance to cisplatin in lung cancer stem cells. Cancer Lett 406: 93-104, 2017.

18. Noto A, De Vitis C, Pisanu ME, Roscilli G, Ricci G, Catizone A, Sorrentino G, Chianese G, Taglialatela-Scafati O, Trisciuoglio D, et al: Stearoyl-CoA-desaturase 1 regulates lung cancer stemness via stabilization and nuclear localization of YAP/TAZ. Oncogene 36: 4573-4584, 2017.

19. Tao T, Su Q, Xu S, Deng J, Zhou S, Zhuang Y, Huang Y, He C, He S, Peng M, et al: Down-regulation of PKM2 decreases FASN expression in bladder cancer cells through AKT/mTOR/SREBP-1c axis. J Cell Physiol 234: 3088-3104, 2019.

20. Zhu J, Jin J, Ding J, Li S, Cen P, Wang K, Wang H and Xia J: Ganoderic Acid A improves high fat diet-induced obesity, lipid accumulation and insulin sensitivity through regulating SREBP pathway. Chem-Biol Interact 290: 77-87, 2018.

21. Olmstead AD, Knecht W, Lazarov I, Dixit SB and Jean F: Human subtilase SKI-1/S1P is a master regulator of the HCV Lifecycle and a potential host cell target for developing indirect-acting antiviral agents. PLoS Pathog 8: e1002468, 2012.

22. Mustafa M, Wang TN, Chen X, Gao B and Krepinsky JC: SREBP inhibition ameliorates renal injury after unilateral ureteral obstruction. Am J Physiol Renal Physiol 311: F614-F625, 2016.

23. Zhou C, Qian W, Ma J, Cheng L, Jiang Z, Yan B, Li J, Duan W, Sun L, Cao J, et al: Resveratrol enhances the chemotherapeutic response and reverses the stemness induced by gemcitabine in pancreatic cancer cells via targeting SREBP1. Cell Prolif 52: e12514, 2019.

24. Nie LY, Lu QT, Li WH, Yang N, Dongol S, Zhang X and Jiang J: Sterol regulatory element-binding protein 1 is required for ovarian tumor growth. Oncol Rep 30: 1346-1354, 2013.

25. Cheng C, Ru P, Geng F, Liu J, Yoo JY, Wu X, Cheng X, Euthine V, Hu P, Guo JY, et al: Glucose-Mediated N-glycosylation of SCAP is essential for SREBP-1 activation and tumor growth. Cancer Cell 28: 569-581, 2015.

26. Griffiths B, Lewis CA, Bensaad K, Ros S, Zhang Q, Ferber EC, Konisti S, Peck B, Miess H, East P, et al: Sterol regulatory element binding protein-dependent regulation of lipid synthesis supports cell survival and tumor growth. Cancer Metab 1: 3, 2013.

27. Singh KB, Hahm ER, Pore SK and Singh SV: Leelamine is a novel lipogenesis inhibitor in prostate cancer cells in vitro and in vivo. Mol Cancer Ther 18: 1800-1810, 2019.

28. Gao Y, Nan X, Shi X, Mu X, Liu B, Zhu H, Yao B, Liu X, Yang T, $\mathrm{Hu}$ Y and Liu S: SREBP1 promotes the invasion of colorectal cancer accompanied upregulation of MMP7 expression and NF-kappaB pathway activation. BMC Cancer 19: 685, 2019.

29. Yoshida GJ, Saya H and Zouboulis CC: Three-dimensional culture of sebaceous gland cells revealing the role of prostaglandin E2-induced activation of canonical Wnt signaling. Biochem Biophys Res Commun 438: 640-646, 2013.

30. Wang Y, Guo D, He J, Song L, Chen H, Zhang Z and Tan N: Inhibition of fatty acid synthesis arrests colorectal neoplasm growth and metastasis: Anti-cancer therapeutical effects of natural cyclopeptide RA-XII. Biochem Biophys Res Commun 512: 819-824, 2019.

31. Sorrentino G, Ruggeri N, Specchia V, Cordenonsi M, Mano M, Dupont S, Manfrin A, Ingallina E, Sommaggio R, Piazza S, et al: Metabolic control of YAP and TAZ by the mevalonate pathway. Nat Cell Biol 16: 357-366, 2014.

32. Bertolio R, Napoletano F, Mano M, Maurer-Stroh S, Fantuz M, Zannini A, Bicciato S, Sorrentino G and Del Sal G: Sterol regulatory element binding protein 1 couples mechanical cues and lipid metabolism. Nat Commun 10: 1326, 2019. 
33. Sodi VL, Bacigalupa ZA, Ferrer CM, Lee JV, Gocal WA Mukhopadhyay D, Wellen KE, Ivan M and Reginato MJ: Nutrient sensor O-GlcNAc transferase controls cancer lipid metabolism via SREBP-1 regulation. Oncogene 37: 924-934, 2018.

34. Du T, Sikora MJ, Levine KM, Tasdemir N, Riggins RB, Wendell SG, Van Houten B and Oesterreich S: Key regulators of lipid metabolism drive endocrine resistance in invasive lobular breast cancer. Breast Cancer Res 20: 106, 2018.

35. Zhou C, Qian W, Li J, Ma J, Chen X, Jiang Z, Cheng L, Duan W, Wang Z, Wu Z, et al: High glucose microenvironment accelerates tumor growth via SREBP1-autophagy axis in pancreatic cancer. J Exp Clin Cancer Res 38: 302, 2019.

36. Gouw AM, Margulis K, Liu NS, Raman SJ, Mancuso A, Toal GG, Tong L, Mosley A, Hsieh AL, Sullivan DK, et al: The MYC oncogene cooperates with sterol-regulated element-binding protein to regulate lipogenesis essential for neoplastic growth. Cell Metab 30: 556-572.e5, 2019.

37. Hu N, Clifford RJ, Yang HH, Wang C, Goldstein AM, Ding T, Taylor PR and Lee MP: Genome wide analysis of DNA copy number neutral loss of heterozygosity $(\mathrm{CNNLOH})$ and its relation to gene expression in esophageal squamous cell carcinoma. BMC Genomics 11: 576, 2010.

38. Su H, Hu N, Yang HH, Wang C, Takikita M, Wang QH, Giffen C, Clifford R, Hewitt SM, Shou JZ, et al: Global gene expression profiling and validation in esophageal squamous cell carcinoma and its association with clinical phenotypes. Clin Cancer Res 17: 2955-2966, 2011.

39. Hu G, Zhou Y, Zhu Y, Zhou L, Ling R, Wu D, Mi L, Wang X, Dai D, Mao C and Chen D: Novel transduction of nutrient stress to Notch pathway by RasGRP3 promotes malignant aggressiveness in human esophageal squamous cell carcinoma. Oncol Rep 38: 2975-2984, 2017.

40. Zhou Y, Su Y, Zhu H, Wang X, Li X, Dai C, Xu C, Zheng T, Mao $C$ and Chen D: Interleukin-23 receptor signaling mediates cancer dormancy and radioresistance in human esophageal squamous carcinoma cells via the Wnt/Notch pathway. J Mol Med (Berl) 97: 177-188, 2019.

41. Brown M, Hart C, Tawadros T, Ramani V, Sangar V, Lau M and Clarke N: The differential effects of statins on the metastatic behaviour of prostate cancer. Br J Cancer 106: 1689-1696, 2012.

42. Huang CM, Huang CS, Hsu TN, Huang MS, Fong IH, Lee WH and Liu SC: Disruption of cancer metabolic SREBP1/miR-142-5p suppresses epithelial-mesenchymal transition and stemness in esophageal carcinoma. Cells 9: 7, 2019.

43. Zhou C, Zhang L and Xu P: Growth inhibition and chemo-radiosensitization of esophageal squamous cell carcinoma by survivin-shRNA lentivirus transfection. Oncol Lett 16: 4813-4820, 2018.

44. Livak KJ and Schmittgen TD: Analysis of relative gene expression data using real-time quantitative PCR and the 2(-Delta Delta C(T)) method. Methods 25: 402-408, 2001.

45. Shao W, Machamer CE and Espenshade PJ: Fatostatin blocks ER exit of SCAP but inhibits cell growth in a SCAP-independent manner. J Lipid Res 57: 1564-1573, 2016.

46. Pierrot N, Tyteca D, D'Auria L, Dewachter I, Gailly P, Hendrickx A, Tasiaux B, Haylani LE, Muls N, N'kuli F, et al: Amyloid precursor protein controls cholesterol turnover needed for neuronal activity. EMBO Mol Med 5: 608-625, 2013.

47. Guillet-Deniau I, Pichard AL, Koné A, Esnous C, Nieruchalski M, Girard J and Prip-Buus C: Glucose induces de novo lipogenesis in rat muscle satellite cells through a sterol-regulatory-element-bindi ng-protein-1c-dependent pathway. J Cell Sci 117: 1937-1944, 2004.

48. Koizume S, Takahashi T, Yoshihara M, Nakamura Y, Ruf W, Takenaka K, Miyagi E and Miyagi Y: Cholesterol starvation and hypoxia activate the FVII gene via the SREBP1-GILZ pathway in ovarian cancer cells to produce procoagulant microvesicles. Thromb Haemost 119: 1058-1071, 2019.
49. Lo AK, Lung RW, Dawson CW, Young LS, Ko CW, Yeung WW, Kang W, To KF and Lo KW: Activation of sterol regulatory element-binding protein 1 (SREBP1)-mediated lipogenesis by the Epstein-Barr virus-encoded latent membrane protein 1 (LMP1) promotes cell proliferation and progression of nasopharyngeal carcinoma. J Pathol 246: 180-190, 2018.

50. Perone Y, Farrugia AJ, Meira AR, Rodríguez-Meira A, Győrffy B, Ion C, Uggetti A, Chronopoulos A, Marrazzo P, Faronato M, et al: SREBP1 drives Keratin-80-dependent cytoskeletal changes and invasive behavior in endocrine-resistant ER $\alpha$ breast cancer. Nat Commun 10: 2115, 2019.

51. Zhang N, Zhang H, Liu Y, Su P, Zhang J, Wang X, Sun M, Chen B, Zhao W, Wang L, et al: SREBP1, targeted by miR-18a-5p, modulates epithelial-mesenchymal transition in breast cancer via forming a co-repressor complex with Snail and HDAC1/2. Cell Death Differ 26: 843-859, 2019.

52. Yang J, Antin P, Berx G, Blanpain C, Brabletz T, Bronner M, Campbell K, Cano A, Casanova J, Christofori G, et al: Guidelines and definitions for research on epithelial-mesenchymal transition. Nat Rev Mol Cell Biol 21: 341-352, 2020.

53. Gong F, Chen MF, Chen J, Li C, Zhou C, Hong P, Sun S and Qian ZJ: Boiled abalone byproduct peptide exhibits anti-tumor activity in HT1080 cells and HUVECs by suppressing the metastasis and angiogenesis in vitro. J Agric Food Chem 67: 8855-8867, 2019.

54. Motoyama K, Fukumoto S, Koyama H, Emoto M, Shimano H, Maemura K and Nishizawa Y: SREBP inhibits VEGF expression in human smooth muscle cells. Biochem Biophys Res Commun 342: 354-360, 2006.

55. Zhang L, Wang JH, Liang RX, Huang ST, Xu J, Yuan LJ, Huang L, Zhou Y, Yu XJ, Wu SY, et al: RASSF8 downregulation promotes lymphangiogenesis and metastasis in esophageal squamous cell carcinoma. Oncotarget 6: 34510-34524, 2015.

56. Liu G, Kuang S, Cao R, Wang J, Peng Q and Sun C: Sorafenib kills liver cancer cells by disrupting SCD1-mediated synthesis of monounsaturated fatty acids via the ATP-AMPK-mTOR-SREBP1 signaling pathway. FASEB J 33: 10089-10103, 2019.

57. Jain $P$, Nattakom M, Holowka D, Wang DH, Thomas Brenna J, $\mathrm{Ku}$ AT, Nguyen H, Ibrahim SF and Tumbar T: Runx1 role in epithelial and cancer cell proliferation implicates lipid metabolism and scd1 and soatl Activity. Stem cells 36: 1603-1616, 2018.

58. Mancini R, Noto A, Pisanu ME, De Vitis C, Maugeri-Sacca M and Ciliberto G: Metabolic features of cancer stem cells: The emerging role of lipid metabolism. Oncogene 37: 2367-2378, 2018.

59. El Helou R, Pinna G, Cabaud O, Wicinski J, Bhajun R, Guyon L, Rioualen C, Finetti P, Gros A, Mari B, et al: miR-600 acts as a bimodal switch that regulates breast cancer stem cell fate through WNT signaling. Cell Rep 18: 2256-2268, 2017.

60. Bruschini S, di Martino S, Pisanu ME, Fattore L, De Vitis C, Laquintana V, Buglioni S, Tabbì E, Cerri A, Visca $\mathrm{P}$, et al: CytoMatrix for a reliable and simple characterization of lung cancer stem cells from malignant pleural effusions. J Cell Physiol 235: 1877-1887, 2020.

61. Lim CH, Park YJ, Shin M, Cho YS, Choi JY, Lee KH and Hyun SH: Tumor SUVs on 18F-FDG PET/CT and aggressive pathological features in esophageal squamous cell carcinoma. Clin Nucl Med 45: e128-e133, 2020.

This work is licensed under a Creative Commons Attribution-NonCommercial-NoDerivatives 4.0 International (CC BY-NC-ND 4.0) License. 